JOURNAL OF THE

AMERICAN MATHEMATICAL SOCIETY

Volume 19, Number 1, Pages 181-204

S 0894-0347(05)00497-2

Article electronically published on July 12, 2005

\title{
POSITIVITY OF QUASI-LOCAL MASS II
}

\author{
CHIU-CHU MELISSA LIU AND SHING-TUNG YAU
}

\section{INTRODUCTION}

A spacetime is a four-manifold with a pseudo-metric of signature $(+,+,+,-)$. A hypersurface or a 2-surface in a spacetime is spacelike if the induced metric is positive definite. A quasi-local energy-momentum vector is a vector in $\mathbb{R}^{3,1}$ associated to a spacelike 2-surface which depends on its first and second fundamental forms and the connection to its normal bundle in the spacetime. The time component of the four-vector is called quasi-local energy (mass). Similar to [10, 8, we require the quasi-local energy-momentum vector to satisfy the following properties.

(1) It should be zero for the flat spacetime.

(2) The quasi-local mass should be equivalent to the standard definition if the spacetime is spherically symmetric and the quasi-local mass is evaluated on the spheres [7. (We say that two masses $m_{1}$ and $m_{2}$ are equivalent if there is a universal constant $c>0$ such that $c^{-1} m_{1} \leq m_{2} \leq c m_{1}$.) In particular, for the centered spheres in the Schwarzschild spacetime, the quasi-local mass should be equivalent to the standard mass.

(3) For an asymptotically flat slice, the quasi-local mass of the coordinate sphere should be asymptotic to the ADM energy-momentum vector.

(4) For an asymptotically null slice, the quasi-local mass of the coordinate sphere should be asymptotic to the Bondi energy-momentum vector.

(5) For an apparent horizon $\Sigma$, the quasi-local mass should be no less than a (universal) constant multiple of the irreducible mass, which is $\sqrt{\operatorname{Area}(\Sigma) / 16 \pi}$.

(6) The quasi-local energy-momentum vector should be nonspacelike and the quasi-local mass should be nonnegative.

Our definition of quasi-local energy [22] arises naturally from calculations in the second author's work [36] on black holes and is strongly motivated by our ability to prove its positivity. After the second author proposed our definition, we were informed of the existence of much earlier works by Brown-York [3, 4] and others 21, 20, 11. The main goal of this paper is to provide a complete proof of a stronger version of the positivity stated in [22].

The rest of the paper is organized as follows. In Section 2, we recall our definition of quasi-local energy, discuss its properties, and state the main result (positivity of

Received by the editors January 20, 2005.

2000 Mathematics Subject Classification. Primary 83C99; Secondary 53C21, 53C27.

Key words and phrases. Quasi-local energy, local energy condition, positive mass theorem.

The second author is supported in part by the National Science Foundation under Grant No. DMS-0306600.

(C)2005 by Chiu-Chu Melissa Liu and Shing-Tung Yau 
quasi-local energy). In Section 3 we describe Shi and Tam's proof of positivity in the Riemannian case. In Section 4 , we prove the main result.

\section{Definition of QUASI-LOCAL ENERGy AND ITS PROPERTIES}

Let $\Sigma$ be a spacelike 2 -surface in a spacetime $N$. At each point of $\Sigma$, choose two null normals $l, n$ such that $\langle l, n\rangle=-1$. Any other choice $\left(l^{\prime}, n^{\prime}\right)$ is related to $(l, n)$ by $l^{\prime}=\lambda l, n^{\prime}=\lambda^{-1} n$ or $l^{\prime}=\lambda n, n^{\prime}=\lambda^{-1} l$ for some function $\lambda: \Sigma \rightarrow \mathbb{R} \backslash\{0\}$. We denote the mean curvature with respect to $l$ and $n$ by

$$
2 \rho=-\left\langle\nabla_{1} e_{1}+\nabla_{2} e_{2}, l\right\rangle, \quad-2 \mu=-\left\langle\nabla_{1} e_{1}+\nabla_{2} e_{2}, n\right\rangle
$$

respectively, where $\left\{e_{1}, e_{2}\right\}$ is a local orthonormal frame of $\Sigma$. The definitions of $\rho$ and $\mu$ depend on the choice of $(l, n)$, but their product $\rho \mu$ is independent of the choice of $(l, n)$. More intrinsically,

$$
8 \rho \mu=\langle\mathbf{H}, \mathbf{H}\rangle,
$$

where $\mathbf{H}$ is the mean curvature vector of $\Sigma$ in $N$. We assume that $\rho \mu>0$, or equivalently, the mean curvature vector $\mathbf{H}$ of $\Sigma$ in $N$ is spacelike.

Suppose that $\Sigma$ has positive Gaussian curvature so that $\Sigma$ is topologically a 2sphere. By Weyl's embedding theorem, $\Sigma$ can be isometrically embedded into the Euclidean space $\mathbb{R}^{3}$ so that the second fundamental form $\left(H_{0}\right)_{a b}$ is positive definite. The embedding $\Sigma \subset \mathbb{R}^{3}$ is unique up to an isometry of $\mathbb{R}^{3}$, so $\left(H_{0}\right)_{a b}$ is determined by the metric on $\Sigma$. Let $\rho_{0}, \mu_{0}$ be the mean curvatures with respect to null normals $l_{0}, n_{0}$ of the embedding $\Sigma \subset \mathbb{R}^{3} \subset \mathbb{R}^{3,1}$, with the normalization $\left\langle l_{0}, n_{0}\right\rangle=-1$. Then

$$
8 \rho_{0} \mu_{0}=H_{0}^{2},
$$

where $H_{0}>0$ is the trace of $\left(H_{0}\right)_{a b}$. Define the quasi-local energy of $\Sigma$ to be

$$
E(\Sigma)=\frac{1}{8 \pi G} \int_{\Sigma}\left(\sqrt{8 \rho_{0} \mu_{0}}-\sqrt{8 \rho \mu}\right)=\frac{1}{8 \pi G} \int_{\Sigma}\left(H_{0}-\sqrt{8 \rho \mu}\right) .
$$

See 33 for other definitions of quasi-local energy.

Recall (1)-(6) in Section 1] In [26, Murchadha, Szabados, and Tod gave examples of $\Sigma \subset \mathbb{R}^{3,1}$ but $E(\Sigma)>0$, so $E(\Sigma)$ does not satisfy (1). For (2), recall that the Schwarzschild spacetime metric on $\mathbb{R}^{4}$ is given by

$$
g=-\left(1-\frac{2 M}{r}\right) d t^{2}+\left(1-\frac{2 M}{r}\right)^{-1} d r^{2}+r^{2}\left(d \theta^{2}+\sin ^{2} \theta d \phi^{2}\right), \quad r>2 M,
$$

where $r, \theta, \phi$ are the spherical coordinates on $\mathbb{R}^{3}$. Let $S_{a} \subset\left(\mathbb{R}^{4}, g\right)$ be the round sphere defined by $t=0, r=a$, and let $m(r)=E\left(S_{r}\right)$. Then

$$
m(r)=r\left(1-\sqrt{1-\frac{2 M}{r}}\right) .
$$

Note that $m(r)$ is decreasing (for $r \geq 2 M$ ), $m(2 M)=2 M$, and $m(\infty)=M$, which is consistent with (2). For (3), (4), Epp discussed the spatial and future null infinity limits of a large sphere in asymptotically flat spacetimes, but cannot conclude that $E(\Sigma)$ satisfies $(3),(4)$ in general. For (5), on an apparent horizon $\Sigma$ we have $\rho \mu=0$, so

$$
E(\Sigma)=\frac{1}{8 \pi} \int_{\Sigma} H_{0} \geq \sqrt{\frac{\operatorname{Area}(\Sigma)}{4 \pi}}
$$


by the Minkowski inequality of convex bodies [24. Therefore, $E(\Sigma)$ satisfies (5). By the main result of this paper, $E(\Sigma)$ is nonnegative as required in (6) and is strictly positive when the spacetime is not flat along $\Sigma$.

We now give a precise statement of the main result. Let $\Omega$ be a compact spacelike hypersurface in a time-orientable four-dimensional spacetime $N$. Let $g_{i j}$ denote the induced metric on $\Omega$, and let $p_{i j}$ denote the second fundamental form of $\Omega$ in $N$. The local mass density $\mu$ and the local current density $J^{i}$ on $\Omega$ are related to $g_{i j}$ and $p_{i j}$ by the constraint equations

$$
\begin{aligned}
\mu & =\frac{1}{2}\left(R-\sum_{i, j} p^{i j} p_{i j}+\left(\sum_{i} p_{i}^{i}\right)^{2}\right), \\
J^{i} & =\sum_{j} D_{j}\left(p^{i j}-\left(\sum_{k} p_{k}^{k}\right) g^{i j}\right),
\end{aligned}
$$

where $R$ is the scalar curvature of the metric $g_{i j}$. In this paper, we prove the following stronger version of the positivity stated in 22 .

Theorem 1 (positivity of quasi-local energy). Let $\Omega, \mu, J$ be as above. We assume that $\mu$ and $J^{i}$ satisfy the local energy condition

$$
\mu \geq \sqrt{J^{i} J_{i}}
$$

and that the boundary $\partial \Omega$ has finitely many connected components $\Sigma^{1}, \ldots, \Sigma^{\ell}$, each of which has positive Gaussian curvature and has spacelike mean curvature vector in $N$. Let $E\left(\Sigma^{\alpha}\right)$ be defined as in (2). Then $E\left(\Sigma^{\alpha}\right) \geq 0$ for $\alpha=1, \ldots, \ell$. Moreover, if $E\left(\Sigma^{\alpha}\right)=0$ for some $\alpha$, then $M$ is flat spacetime along $\Omega$, and $\partial \Omega$ is connected and will be embedded into $\mathbb{R}^{3} \subset \mathbb{R}^{3,1}$ by the well-known Weyl embedding theorem.

\section{The Riemannian Case}

When the second fundamental form of $\Omega$ in $N$ vanishes, the local energy condition (5) reduces to $R \geq 0$ and the condition $\rho \mu>0$ reduces to $H>0$, where $H$ is the mean curvature of the spacelike 2 -surface in $\Omega$ with respect to the outward unit normal. Shi and Tam proved positivity of quasi-local energy in this case.

Theorem 2 ([31, Theorem 1]). Let $\left(\Omega^{3}, g\right)$ be a compact manifold of dimension three with smooth boundary and with nonnegative scalar curvature. Suppose $\partial \Omega$ has finitely many connected components $\Sigma^{\alpha}$ so that each connected component has positive Gaussian curvature and positive mean curvature $H$ with respect to the unit outward normal. Then for each boundary component $\Sigma^{\alpha}$,

$$
\int_{\Sigma^{\alpha}} H d \sigma \leq \int_{\Sigma^{\alpha}} H_{0}^{\alpha} d \sigma
$$

where $H_{0}^{\alpha}$ is the mean curvature of $\Sigma^{\alpha}$ with respect to the outward normal when it is isometrically embedded in $\mathbb{R}^{3}$ and $d \sigma$ is the volume form on $\Sigma^{\alpha}$ induced from g. Moreover, if equality holds in (6) for some $\Sigma^{\alpha}$, then $\partial \Omega$ has only one connected component and $\Omega$ is a domain in $\mathbb{R}^{3}$.

We now briefly describe Shi and Tam's proof of Theorem 2, From now on, all the mean curvatures are defined with respect to the outward unit normal.

Let $\Sigma^{\alpha}$ be a connected component of $\partial \Omega$. By the hypothesis of Theorem 2, it has positive Gaussian curvature, so it can be isometrically embedded in $\mathbb{R}^{3}$ by the 
well-known Weyl embedding theorem. Moreover, the embedding is unique up to an isometry of $\mathbb{R}^{3}$. Let $\Sigma_{0}^{\alpha} \subset \mathbb{R}^{3}$ be the image of such an embedding. Then $\Sigma_{0}^{\alpha}$ is a strictly convex hypersurface diffeomorphic to $S^{2}$.

Let $\mathbf{X}$ be the position vector of a point on $\Sigma_{0}^{\alpha}$, and let $\mathbf{N}$ be the unit outward normal of $\Sigma_{0}^{\alpha}$ at $\mathbf{X}$. Let $\Sigma_{r}^{\alpha}$ be the surface described by $\mathbf{Y}=\mathbf{X}+r \mathbf{N}$, with $r \geq 0$. Let $D^{\alpha}$ be the region of $\mathbb{R}^{3}$ outside $\Sigma_{0}^{\alpha}$, and let $E^{\alpha}=D^{\alpha} \cup \Sigma_{0}^{\alpha}$ be the closure of $D^{\alpha}$ in $\mathbb{R}^{3}$. Then $E^{\alpha}$ can be represented by

$$
\left(\Sigma^{\alpha} \times[0, \infty), g^{0}=d r^{2}+g_{r}\right),
$$

where $g_{r}$ is the induced metric on $\Sigma_{r}^{\alpha}$ and $g^{0}=d r^{2}+g_{r}$ is the standard Euclidean metric on $E^{\alpha} \subset \mathbb{R}^{3}$. Note that $g_{r}=(a+r)^{2}\left(d \theta^{2}+\sin ^{2} \theta d \phi^{2}\right)$ if $\Sigma_{0}^{\alpha}$ is a round sphere of radius $a>0$.

Consider a Riemannian metric on $E^{\alpha}$ of the form

$$
g=h^{2} d r^{2}+g_{r},
$$

where $h$ is a smooth positive function. This is a special case of Bartnik's construction in [2]. Note that $g$ and $g^{0}$ induce the same metric on each $\Sigma_{r}^{\alpha}$. The mean curvatures $H$ and $H_{0}$ of $\Sigma_{r}^{\alpha}$ with respect to $g$ and $g^{0}$ are related by

$$
H=h^{-1} H^{0} \text {. }
$$

Note that $H_{0}(x, 0)=H_{0}^{\alpha}(x)$ for $x \in \Sigma_{0}^{\alpha} \cong \Sigma^{\alpha}$. The scalar curvature $R$ of $g$ is given by

$$
2 H_{0} \frac{\partial h}{\partial r}=2 h^{2} \Delta_{r} h+\left(h-h^{3}\right) R^{r}+h^{3} R,
$$

where $R^{r}$ is the scalar curvature of $\Sigma_{r}^{\alpha}$, and $\Delta_{r}$ is the Laplacian operator on $\Sigma_{r}^{\alpha}$. So a solution to the parabolic partial differential equation

$$
2 H_{0} \frac{\partial h}{\partial r}=2 h^{2} \Delta_{r} h+\left(h-h^{3}\right) R^{r}
$$

on $E^{\alpha} \cong \Sigma^{\alpha} \times[0, \infty)$ with the initial condition

$$
h(x, 0)=\frac{H_{0}^{\alpha}}{H}
$$

defines a metric on $E^{\alpha}$ such that the scalar curvature $R=0$ and the mean curvature of $\Sigma_{0}^{\alpha}$ coincides with the restriction of $H$ to $\Sigma^{\alpha} \cong \Sigma_{0}^{\alpha}$.

Let $\rho: \mathbb{R}^{3} \rightarrow[0, \infty)$ be the distance function to the origin (in the Euclidean metric). We may assume that the origin is enclosed by $\Sigma_{0}^{\alpha}$ so that $\rho \geq a$ for some constant $a>0$. Shi and Tam showed that [31, Theorem 2.1]:

Theorem 3. The equation (8) with the initial condition (9) has a unique solution such that

(a) $h=1+m_{o} \rho^{-1}+\kappa$, where $m_{0}$ is a constant and the function $\kappa$ satisfies

$$
|\kappa|=O\left(\rho^{-2}\right), \quad\left|\nabla_{0} \kappa\right|=O\left(\rho^{-3}\right) .
$$

Here $\nabla_{0}$ is the Levi-Civita connection of the Euclidean metric on $\mathbb{R}^{3}$.

(b) The metric $g^{\alpha}=h^{2} d r^{2}+g_{r}$ on $E^{\alpha}$ is asymptotically flat in the sense that

$$
\left|g_{i j}^{\alpha}-\delta_{i j}\right|+\rho\left|\nabla_{0} g_{i j}^{\alpha}\right|+\rho^{2}\left|\nabla_{0}^{2} g_{i j}^{\alpha}\right| \leq C \rho^{-1}
$$

with zero scalar curvature. 
(c) The ADM mass of $\left(E^{\alpha}, g^{\alpha}\right)$ is given by

$$
m_{\infty}^{\alpha}=\lim _{r \rightarrow \infty} m^{\alpha}(r)
$$

where

$$
m^{\alpha}(r)=\frac{1}{8 \pi G} \int_{\Sigma_{r}^{\alpha}}\left(H_{0}-H\right) d \sigma_{r}
$$

and $d \sigma_{r}$ is the volume form of $\Sigma_{r}^{\alpha}$.

Let $m^{\alpha}(r)$ be defined as in (c) of Theorem 3, It is computed in the proof of 31, Lemma 4.2] that

$$
\frac{d m^{\alpha}}{d r}(r)=\frac{-1}{16 \pi G} \int_{\Sigma_{r}^{\alpha}} R^{r} u^{-1}(1-u)^{2} \leq 0 .
$$

Shi and Tam glued $\left(E^{\alpha}, g^{\alpha}\right)$ to $(\Omega, g)$ along $\Sigma^{\alpha}$ to obtain a complete noncompact three manifold $M$ with a continuous Riemannian metric $\tilde{g}$ such that

(1) $\tilde{g}$ is smooth on $M \backslash \Omega$ and $\Omega$ and is Lipschitz near $\partial \Omega$.

(2) The mean curvatures of $\Sigma^{\alpha}$ with respect to $g=\left.\tilde{g}\right|_{\Omega}$ and $g^{\alpha}=\left.\tilde{g}\right|_{E^{\alpha}}$ are the same for each $\alpha$.

(3) Each end $E^{\alpha}$ of $M$ is asymptotically Euclidean in the sense of (10).

(4) The scalar curvature $R$ of $M \backslash \partial \Omega$ is nonnegative and is in $L^{1}(M)$.

Using Witten's argument [35, 27, Shi and Tam proved that the positive mass theorem holds for such a metric, so the ADM mass

$$
m_{\infty}^{\alpha}=\lim _{r \rightarrow \infty} m^{\alpha}(r)
$$

is nonnegative for each end $E^{\alpha}$, and $m_{\infty}^{\alpha}$ vanishes for some $\alpha$ if and only if $M$ has only one end and $M$ is flat. This together with the monotonicity (11) of $m^{\alpha}(r)$ gives Theorem 2, since

$$
m^{\alpha}(0)=\frac{1}{8 \pi G} \int_{\Sigma^{\alpha}}\left(H_{0}^{\alpha}-H\right) d \sigma .
$$

\section{Proof of Theorem 1}

4.1. Outline of proof. Let $\left(\Omega, g_{i j}, p_{i j}\right)$ and $\Sigma^{1}, \ldots, \Sigma^{\ell}$ be as in Section 2. We first deform the metric $g_{i j}$ on $\Omega$ by a procedure used by Schoen and the second author in 32 and also by the second author in [36. This procedure consists of two steps. The first step is to deform $g_{i j}$ to a new metric

$$
\bar{g}_{i j}=g_{i j}+f_{i} f_{j}
$$

where $f$ is a solution to Jang's equation on $\Omega$ such that $\left.f\right|_{\partial \Omega}=0$. The metric $\bar{g}_{i j}$ coincides with $g_{i j}$ when restricted to $\partial \Omega$, and its scalar curvature $\bar{R}$ satisfies

$$
\bar{R} \geq 2|X|^{2}-2 \operatorname{div} X
$$

for some vector field $X$ on $\Omega$. The equality holds only if $p_{i j}=h_{i j}$, where $h_{i j}$ is the second fundamental form of the isometric embedding of $\left(\Omega, \bar{g}_{i j}\right)$ into

$$
\left(\Omega \times \mathbb{R}, g_{i j} d x^{i} d x^{j}+d t^{2}\right)
$$

as the graph of $f$. The second step is to deform $\bar{g}_{i j}$ conformally to a metric with zero scalar curvature. The inequality (12) implies that there is a unique metric $\hat{g}_{i j}$ in the conformal class of $\bar{g}_{i j}$ which has zero scalar curvature and coincides with $\bar{g}_{i j}$ on $\partial \Omega$. 
After the above reduction, we cannot apply Theorem 2 directly to $\hat{g}_{i j}$ because the mean curvature $\hat{H}$ of $\partial \Omega$ with respect to $\hat{g}_{i j}$ is not necessarily positive (this point was overlooked in [22]). Instead, we have

$$
\int_{\partial \Omega} \hat{H} \geq \int_{\partial \Omega}(\bar{H}-\langle X, \bar{\nu}\rangle)
$$

where $\bar{H}$ and $\bar{\nu}$ are the mean curvature and outward unit normal of $\partial \Omega$ with respect to $\bar{g}_{i j}$, and the equality holds iff $\hat{g}=\bar{g}$ and $X=0$ (so $p_{i j}=h_{i j}$ ). It was shown in 36 that

$$
\bar{H}-\langle X, \bar{\nu}\rangle \geq \sqrt{H^{2}-P^{2}}=\sqrt{8 \rho \mu},
$$

where $P$ is the trace of the restriction of $p_{i j}$ to $\partial \Omega$. In particular, $\bar{H}-\langle X, \bar{\nu}\rangle$ is positive.

Let $E^{\alpha}$ and $H_{0}^{\alpha}$ be defined as in Section 3 Shi and Tam's proof of Theorem 3 shows that one can solve (8) on $E^{\alpha}$ with the initial condition

$$
h(x, 0)=\frac{H_{0}^{\alpha}}{\bar{H}-\langle X, \bar{\nu}\rangle}
$$

and obtain a scalar flat, asymptotically flat metric $g^{\alpha}$ on the end $E^{\alpha}$. Gluing $\left(E^{\alpha}, g^{\alpha}\right)$ to $(\Omega, \hat{g})$ along $\Sigma^{\alpha}$, we obtain a complete noncompact three manifold $M$ with a Lipschitz continuous Riemannian metric $\tilde{g}$. On $M \backslash \partial \Omega, \tilde{g}$ is smooth and has zero scalar curvature. However, the mean curvatures of $\Sigma^{\alpha}$ with respect to $\hat{g}=\left.\tilde{g}\right|_{\Omega}$ and $g^{\alpha}=\left.g\right|_{E^{\alpha}}$ are not necessarily the same. This causes the following problem which is absent in the case considered by Shi and Tam: the zeroth-order term of the Dirac operator can be discontinuous along $\partial \Omega$, so there is an extra term when we integrate the Weitzenböck-Lichnerowicz formula. To prove the positive mass theorem for $(M, \tilde{g})$ (Theorem 7), we derive an inequality (Proposition 10) as a substitute of the integral form of the Weitzenböck-Lichnerowicz formula for smooth metrics.

Let $m_{\infty}^{\alpha}$ and $m^{\alpha}(r)$ be defined by $g^{\alpha}$ as in Section 3 . The monotonicity (111) of $m^{\alpha}(r)$ and (14) imply

$$
m_{\infty}^{\alpha} \leq \frac{1}{8 \pi G} \int_{\Sigma^{\alpha}}\left(H_{0}^{\alpha}-(\bar{H}-\langle X, \bar{\nu}\rangle)\right) \leq \frac{1}{8 \pi G} \int_{\Sigma^{\alpha}}\left(H_{0}^{\alpha}-\sqrt{8 \rho \mu}\right)=E\left(\Sigma^{\alpha}\right) .
$$

The positive mass theorem for $(M, \tilde{g})$ says that $m_{\infty}^{\alpha} \geq 0$ for $\alpha=1, \ldots, \ell$, and $m_{\infty}^{\alpha}=0$ for some $\alpha$ iff $\ell=1$ and $(M, \tilde{g})$ is the Euclidean space $\mathbb{R}^{3}$. So $E\left(\Sigma^{\alpha}\right) \geq 0$ for $\alpha=1, \ldots, \ell$. If $E\left(\Sigma^{\alpha}\right)=0$ for some $\alpha$, we must have $m_{\infty}^{\alpha}=0$ and $\hat{g}=\bar{g}$, so $(\Omega, \bar{g})=(\Omega, \tilde{g})$ is a domain $\Omega_{0} \subset \mathbb{R}^{3}$. In this case, $(\Omega, g)$ (at least the part away from apparent horizons) can be isometrically embedded in $\mathbb{R}^{3,1}=\left(\mathbb{R}^{3} \times \mathbb{R}, \sum d x_{i}^{2}-d t^{2}\right)$ as a graph

$$
\left.\left\{(x, f(x)) \mid x \in \Omega_{0}\right)\right\}
$$

with second fundamental form $p_{i j}$, where $f$ is a smooth function on $\Omega_{0}$ which vanishes on $\partial \Omega_{0}$.

4.2. Jang's equation with Dirichlet boundary condition. As in 32, we consider the following equation proposed by Jang [19] on $\Omega$ :

$$
\sum_{i, j=1}^{3}\left(g^{i j}-\frac{f^{i} f^{j}}{1+|\nabla f|^{2}}\right)\left(\frac{f_{i j}}{\sqrt{1+|\nabla f|^{2}}}-p_{i j}\right)=0 .
$$


As in [36, we consider solutions to (17) with the Dirichlet boundary condition

$$
\left.f\right|_{\partial \Omega} \equiv 0 .
$$

Most of the estimates were made in 32 . To solve the boundary value problem, the second author constructed a barrier in [36] and concluded that there exists a solution to (17) with boundary value (18) when $\left(\Omega, g_{i j}, p_{i j}\right)$ has no apparent horizon.

Definition 4. Let $\left(\Omega, g_{i j}, p_{i j}\right)$ be an initial data set. Given a smooth compact surface $S$ embedded in $\Omega$, let $H_{s}$ be the mean curvature of $S$ with respect to the outward unit normal vector, and let $P_{s}$ be the trace of the restriction of $p_{i j}$ to $S$. A smooth 2-sphere $S$ embedded in $\Omega$ is an apparent horizon of the initial data $\left(\Omega, g_{i j}, p_{i j}\right)$ if $H_{s}+P_{s}=0$ or $H_{s}-P_{s}=0$.

We first assume that $\left(\Omega, g_{i j}, p_{i j}\right)$ has no apparent horizon so that there exists a solution $f$ to Jang's equation (17) on $\Omega$ such that $\left.f\right|_{\partial \Omega}=0$. The induced metric of the graph $\Omega_{f} \cong \Omega$ of $f$ in $\left(\Omega \times \mathbb{R}, g_{i j} d x^{i} d x^{j}+d t^{2}\right)$ is

$$
\bar{g}_{i j}=g_{i j}+f_{i} f_{j}
$$

which can be viewed as a deformation of the metric $g_{i j}$ on $\Omega$. Note that the new metric $\bar{g}$ coincides with the old metric $g$ when restricted to $\partial \Omega$.

We now introduce some notation. Let $\bar{e}_{4}$ be the downward unit normal to $\Omega_{f}$ in $\Omega \times \mathbb{R}$, and let $\bar{e}_{1}, \bar{e}_{2}, \bar{e}_{3}$ be a local orthonormal frame of $\Omega$. We define $h_{i 4}$ by

$$
\bar{\nabla}_{4} \bar{e}_{4}=h_{i 4} \bar{e}_{i}
$$

where $\bar{\nabla}$ denotes the Levi-Civita connection of the metric $g_{i j} d x^{i} d x^{j}+d t^{2}$ on $\Omega \times \mathbb{R}$. Let $h_{i j}=\left\langle\bar{e}_{i}, \bar{\nabla}_{j} \bar{e}_{4}\right\rangle$ be the second fundamental form of $\Omega_{f}$ in $\Omega \times \mathbb{R}$. Let $\bar{R}$ be the scalar curvature of $\bar{g}$, and extend $p_{i j}, \mu, J^{i}$ parallel along the $\mathbb{R}$ factor. The following inequality was derived in [32]:

$$
2(\mu-|J|) \leq \bar{R}-\sum_{i, j}\left(h_{i j}-p_{i j}\right)^{2}-2\left(h_{i 4}-p_{i 4}\right)^{2}+2 \sum_{i} D_{i}\left(h_{i 4}-p_{i 4}\right),
$$

where $D_{i}$ denotes the covariant derivative of $\bar{g}$. In particular,

$$
\bar{R} \geq 2|X|^{2}-2 \operatorname{div} X,
$$

where $X=\sum\left(h_{i 4}-p_{i 4}\right) e_{i}$, and the divergence is defined by $\bar{g}$. By (19), the inequality (201) is an equality only if $p_{i j}=h_{i j}$.

In general, the solution $f$ and the metric $\bar{g}$ are defined on $\Omega^{\prime}$, the complement of the union of apparent horizons, but one can extend $\bar{g}$ to a metric on $\Omega^{\prime \prime}$ which is obtained by adding a point on each end of $\Omega^{\prime}$. See [32] for details.

4.3. Scalar flat metric on $\Omega$. We shall prove the following:

Proposition 5. Let $(\Omega, \bar{g})$ be a compact Riemannian manifold of dimension three with smooth boundary. Suppose that the scalar curvature $\bar{R}$ of $\bar{g}$ satisfies

$$
\bar{R} \geq c|X|^{2}-2 \operatorname{div} X,
$$

for some constant $c>\frac{1}{2}$ and some smooth vector field $X$ on $\Omega$. Then there is a unique metric $\hat{g}_{i j}$ on $\Omega$ such that

(1) the metric $\hat{g}_{i j}$ is conformal to $\bar{g}_{i j}$;

(2) the scalar curvature of $\hat{g}_{i j}$ is zero;

(3) the metric $\hat{g}_{i j}$ coincides with $\bar{g}_{i j}$ on $\partial \Omega$. 
(4) Let $\bar{H}$ and $\hat{H}$ denote the mean curvatures with respect to the metric $\bar{g}$ and $\hat{g}$, respectively, and let $\bar{\nu}$ denote the outward unit normal of $\partial \Omega$ in $(\Omega, \bar{g})$. Then

$$
\int_{\partial \Omega} \hat{H} \geq \int_{\partial \Omega}(\bar{H}-\langle X, \bar{\nu}\rangle)
$$

where the equality holds if and only if $\bar{R}=0, X=0$, and $\hat{g}_{i j}=\bar{g}_{i j}$.

Proof. In this proof, the Laplacian, gradient, divergence, and all the norms are defined by the metric $\bar{g}_{i j}$.

Any metric $\hat{g}_{i j}$ conformal to $\bar{g}_{i j}$ can be written as $\hat{g}_{i j}=u^{4} \bar{g}_{i j}$, where $u$ is a positive smooth function on $\Omega$. The metric $\hat{g}_{i j}$ satisfies (1) and (2) in Proposition 5 if and only if $v=u-1$ is a solution to

$$
\left\{\begin{array}{cl}
\Delta v-\frac{1}{8} \bar{R} v=\frac{1}{8} \bar{R} & \text { on } \Omega, \\
v=0 & \text { on } \partial \Omega .
\end{array}\right.
$$

We first show that (21) has a unique solution. Let $f$ be a solution to

$$
\left\{\begin{array}{cl}
\Delta f-\frac{1}{8} \bar{R} f=0 & \text { on } \Omega \\
f=0 & \text { on } \partial \Omega .
\end{array}\right.
$$

Then

$$
\begin{aligned}
0 & =\int_{\Omega} f\left(-\Delta f+\frac{\bar{R}}{8} f\right)=\int_{\Omega}\left(|\nabla f|^{2}+\frac{\bar{R}}{8} f^{2}\right) \\
& \geq \int_{\Omega}\left(|\nabla f|^{2}+\frac{c}{8}|X|^{2} f^{2}-\frac{1}{4}(\operatorname{div} X) f^{2}\right) \\
& =\int_{\Omega}\left(|\nabla f|^{2}+\frac{1}{2} X(f) f+\frac{c}{8}|X|^{2} f^{2}\right) \\
& \geq \int_{\Omega}\left(|\nabla f|^{2}-\frac{1}{2}|f X||\nabla f|+\frac{c}{8}|f X|^{2}\right) \\
& =\int_{\Omega}\left(\left(\frac{1}{\sqrt{2 c}}|\nabla f|-\frac{\sqrt{2 c}}{4}|f X|\right)^{2}+\left(1-\frac{1}{2 c}\right)|\nabla f|^{2}\right) \\
& \geq 0 .
\end{aligned}
$$

Note that $1-\frac{1}{2 c}>0$, so $\nabla f \equiv 0$, which implies $f \equiv 0$ since $f$ vanishes on $\partial \Omega$. Therefore, zero is the only solution to (22), and (21) has a unique solution. Let $v$ be the unique solution to (21). Then $v$ is smooth.

We next show that $u=v+1$ is positive. Note that $u$ satisfies

$$
\Delta u-\frac{1}{8} \bar{R} u=0
$$

on $\Omega$. Assume that $\Omega_{-}=\{x \in \Omega \mid u(x)<0\}$ is nonempty. Then $\partial \Omega_{-} \cap \partial \Omega=\emptyset$, and

$$
\left\{\begin{array}{cl}
\Delta u-\frac{1}{8} \bar{R} u=0 & \text { on } \Omega_{-}, \\
u=0 & \text { on } \partial \Omega_{-},
\end{array}\right.
$$

which implies that $u \equiv 0$ on $\Omega_{-}$, a contradiction. So $\Omega_{-}$must be empty, or equivalently, $u$ is nonnegative. Since $u=1$ on $\partial \Omega$, the positivity of $u$ follows from the Harnack inequality for nonnegative solutions to (23).

Finally, we check that the metric $\hat{g}_{i j}=u^{4} \bar{g}_{i j}$ satisfies (4) in Proposition 5 . We first compute $\hat{H}$ in terms of $\bar{H}$ and $u$. We may choose coordinates $\left(x^{1}, x^{2}, x^{3}\right)$ near 
a point $p \in \partial \Omega$ such that $\left(x^{1}, x^{2}\right)$ are the normal coordinates of $\partial \Omega$ centered at $p$, and $\frac{\partial}{\partial x^{3}}=\bar{\nu}$. Let $\bar{\Gamma}_{i j}^{k}, \hat{\Gamma}_{i j}^{k}$ denote the Christoffel symbols of $\bar{g}, \hat{g}$, respectively. Then for $i, j=1,2$, at $p$ we have

$$
\begin{aligned}
-\hat{h}_{i j}=\hat{\Gamma}_{i j}^{3} & =\frac{1}{2}\left(\frac{\partial}{\partial x^{i}} \hat{g}_{i 3}+\frac{\partial}{\partial x^{j}} \hat{g}_{j 3}-\frac{\partial}{\partial x^{3}} \hat{g}_{i j}\right) \\
& =\frac{1}{2}\left(\frac{\partial}{\partial x^{i}}\left(u^{4} \bar{g}_{i 3}\right)+\frac{\partial}{\partial x^{j}}\left(u^{4} \bar{g}_{j 3}\right)-\frac{\partial}{\partial x^{3}}\left(u^{4} \bar{g}_{i j}\right)\right) \\
& =\frac{1}{2}\left(\frac{\partial}{\partial x^{i}} \bar{g}_{i 3}+\frac{\partial}{\partial x^{j}} \bar{g}_{j 3}-\frac{\partial}{\partial x^{3}} \bar{g}_{i j}-4 \frac{\partial u}{\partial x^{3}} \delta_{i j}\right) \\
& =\bar{\Gamma}_{i j}^{3}-2 \frac{\partial u}{\partial x^{3}} \delta_{i j} \\
& =-\bar{h}_{i j}-2 \frac{\partial u}{\partial x^{3}} \delta_{i j} .
\end{aligned}
$$

So $\hat{H}=\bar{H}+4 \bar{\nu}(u)$. Also,

$$
\begin{aligned}
\int_{\partial \Omega} \bar{\nu}(u) & =\int_{\Omega} \operatorname{div}(u \nabla u)=\int_{\Omega}\left(|\nabla u|^{2}+u \Delta u\right)=\int_{\Omega}\left(|\nabla u|^{2}+\frac{\bar{R}}{8} u^{2}\right) \\
& \geq \int_{\Omega}\left(|\nabla u|^{2}+\frac{c}{8}|X|^{2} u^{2}-\frac{1}{4} \operatorname{div} X u^{2}\right) \\
& =\int_{\Omega}\left(|\nabla u|^{2}+\frac{c}{8}|X|^{2} u^{2}+\frac{1}{2} X(u) u\right)-\frac{1}{4} \int_{\partial \Omega}\langle X, \bar{\nu}\rangle \\
& \geq \int_{\Omega}\left(\left(\frac{1}{\sqrt{2 c}}|\nabla u|-\frac{\sqrt{2 c}}{4}|u X|\right)^{2}+\left(1-\frac{1}{2 c}\right)|\nabla u|^{2}\right)-\frac{1}{4} \int_{\partial \Omega}\langle X, \bar{\nu}\rangle \\
& \geq-\frac{1}{4} \int_{\partial \Omega}\langle X, \bar{\nu}\rangle .
\end{aligned}
$$

Therefore,

and the equality holds if and only if

$$
\int_{\partial \Omega} \hat{H} \geq \int_{\partial \Omega}(\bar{H}-\langle X, \bar{\nu}\rangle)
$$

$$
u \equiv 1, X=0, \bar{R}=0 .
$$

\subsection{Scalar flat metrics on the ends.}

\section{Lemma 6.}

$$
\bar{H}-\langle X, \bar{\nu}\rangle \geq \sqrt{H^{2}-P^{2}} .
$$

Proof. Let $\left\{\bar{e}_{1}, \bar{e}_{2}, \bar{e}_{3}, \bar{e}_{4}\right\}$ be a local orthonormal frame of $\Omega \times \mathbb{R}$ along the graph $\Omega_{f}$ so that $\bar{e}_{1}, \bar{e}_{2}$ are tangent to $\partial \Omega$ and $\bar{e}_{3}=\bar{\nu}$. Let $w$ be the outward unit normal of $\partial \Omega_{0}$ in $\Omega_{0}$, the graph of the zero function. It was computed in [36, Section 5] that

$$
\bar{H}-\langle X, \bar{\nu}\rangle=-\frac{\left\langle\bar{e}_{4}, w\right\rangle}{\left\langle\bar{e}_{3}, w\right\rangle} P+\frac{1}{\left\langle e_{3}, w\right\rangle} H
$$


Recall that $H>0$, so (24) is equivalent to

$$
\left(-\left\langle\bar{e}_{4}, w\right\rangle P+H\right)^{2} \geq\left\langle\bar{e}_{3}, w\right\rangle^{2}\left(H^{2}-P^{2}\right),
$$

which is equivalent to

$$
\left(\left\langle e_{4}, w\right\rangle^{2}+\left\langle e_{3}, w\right\rangle^{2}\right) P^{2}-2\left\langle e_{4}, w\right\rangle P H+\left(1-\left\langle e_{3}, w\right\rangle^{2}\right) H^{2} \geq 0 .
$$

But $|w|^{2}=1=\left\langle e_{4}, w\right\rangle^{2}+\left\langle e_{3}, w\right\rangle^{2}$, and so this inequality holds trivially.

We now modify Shi-Tam's proof of Theorem 2. Let $\Sigma^{\alpha}, \Sigma_{r}^{\alpha}, E^{\alpha}, g^{\alpha}=h^{2} d r^{2}+g_{r}$ be defined as in Section 3. Shi and Tam's proof in 31 shows that there is a unique solution to (8) on $E^{\alpha} \cong \Sigma^{\alpha} \times[0, \infty)$ with the initial condition

$$
h(x, 0)=\frac{H_{0}^{\alpha}}{\bar{H}-\langle X, \bar{\nu}\rangle}
$$

such that

$$
|h(x, r)-1| \leq \frac{C}{r}
$$

for $r \geq 1$. Equip $E^{\alpha}$ with the metric $g^{\alpha}=h^{2} d r^{2}+g_{r}$. Then $g^{\alpha}$ has zero scalar curvature, and the mean curvature of $\Sigma^{\alpha}$ in $\left(E^{\alpha}, g^{\alpha}\right)$ is $\bar{H}-\langle X, \bar{\nu}\rangle$.

Define

$$
m^{\alpha}(r)=\frac{1}{8 \pi G} \int_{\Sigma_{r}^{\alpha}}\left(H_{0}-H\right) d \sigma_{r}, \quad m_{\infty}^{\alpha}=\lim _{r \rightarrow \infty} m^{\alpha}(r)
$$

as in Section 3, By monotonicity (11), $m_{\infty}^{\alpha} \leq m^{\alpha}(0)$. By Lemma 6]

$m^{\alpha}(0)=\frac{1}{8 \pi G} \int_{\Sigma}\left(H_{0}^{\alpha}-(\bar{H}-\langle X, \bar{\nu}\rangle)\right) d \sigma \leq \frac{1}{8 \pi G} \int_{\Sigma}\left(H_{0}^{\alpha}-\sqrt{H^{2}-P^{2}}\right) d \sigma=E\left(\Sigma^{\alpha}\right)$.

4.5. Asymptotically flat Lipschitz metric. Following [31, we glue $\left(E^{\alpha}, g^{\alpha}\right)$ in Section 4.4 to $(\Omega, \hat{g})$ in Section 4.3 along $\Sigma^{\alpha}$ to obtain a complete noncompact three manifold $M$ with a continuous Riemannian metric $\tilde{g}$ such that

(1) $\tilde{g}$ is smooth on $M \backslash \Omega$ and $\Omega$ and is Lipschitz near $\partial \Omega$;

(2) each end $E^{\alpha}$ of $M$ is asymptotically Euclidean;

(3) the scalar curvature $R$ of $M \backslash \partial \Omega$ is nonnegative and is in $L^{1}(M)$.

The mean curvature of $\Sigma$ with respect to $\hat{g}=\left.\tilde{g}\right|_{\Omega}$ is $\hat{H}=\bar{H}+4 \bar{\nu}(u)$, and the mean curvature of $\Sigma$ with respect to $g^{\alpha}=\left.\tilde{g}\right|_{E^{\alpha}}$ is $\bar{H}-\langle X, \bar{\nu}\rangle$.

Let $\tilde{\nu}$ be the outward unit normal of $\partial \Omega$ with respect to $\tilde{g}$. There exists $\epsilon>0$ such that $(x, t) \mapsto \exp _{x}(t \tilde{\nu}(x))$ defines an open embedding $i: \partial \Omega \times(-\epsilon, \epsilon) \rightarrow M$. The image $T=i(\partial \Omega \times(-\epsilon, \epsilon))$ is a tubular neighborhood of $\partial \Omega$ in $M$. We use the smooth structure on $\partial \Omega \times(-\epsilon, \epsilon)$ to define the smooth structure on $T$. We have

$$
i^{*} \tilde{g}=d t^{2}+\rho_{i j}(x, t) d x_{i} d x_{j}
$$

where $\left(x_{1}, x_{2}\right)$ are local coordinates on $\partial \Omega$.

We choose a local orthonormal frame $e_{1}, e_{2}$ of $\partial \Omega$ and parallel transport them along $\frac{\partial}{\partial t}$. Then $e_{1}, e_{2}$, and $e_{3}=\frac{\partial}{\partial t}$ form a local orthonormal frame on $(\partial \Omega \times$ $\left.(-\epsilon, \epsilon), i^{*} \tilde{g}\right)$ such that

(1) $e_{1}, e_{2}$ are tangent to slides $\Sigma_{t}=\partial \Omega \times\{t\}$;

(2) $e_{3}$ is normal to $\Sigma_{t}$;

(3) $\nabla_{3} e_{i}=0$, where $\nabla$ is the Levi-Civita connection with respect to $i^{*} \tilde{g}$. 
The mean curvature of $\Sigma_{t}$ in $\partial \Omega \times(-\epsilon, \epsilon)$ defines a function $H$ on $\partial \Omega \times(-\epsilon, \epsilon)$ which is discontinuous at $t=0$ and smooth away from $t=0$.

Note that $(M, \tilde{g})$ is uniquely determined by $(\Omega, \hat{g})$, which is uniquely determined by $(\Omega, \bar{g})$. As explained in Section 4.1, our main result Theorem 1 follows from the following positive mass theorem of $(M, \tilde{g})$.

Theorem 7. The ADM mass $m_{\infty}^{\alpha}$ of the end $\left(E^{\alpha}, g^{\alpha}\right)$ is nonnegative for $\alpha=$ $1, \ldots, \ell$, and $m_{\infty}^{\alpha}=0$ for some $\alpha$ if and only if $\ell=1$ and $M$ is the Euclidean space.

We will prove Theorem 7 in Section 4.10 .

4.6. Dirac spinor. In the rest of this paper, $L^{p}, L_{\mathrm{loc}}^{p}, L_{0}^{p}, W^{k, p}, W_{\mathrm{loc}}^{k, p}, W_{0}^{k, p}$ are defined as in [14, Chapter 7].

The spinor bundle $S$ over $M$ is a trivial complex vector bundle of rank 2. Let D : $C^{\infty}(M, S) \rightarrow C^{\infty}(M, S)$ be the Dirac operator defined by the Levi-Civita connection of $\tilde{g}$. It can be extended to $\mathbf{D}: W_{\text {loc }}^{1,2}(M, S) \rightarrow L_{\text {loc }}^{2}(M, S)$.

Let $T$ be the tubular neighborhood of $\partial \Omega$ in $M$ defined in Section 4.5. We identify $T$ with $\partial \Omega \times(-\epsilon, \epsilon)$ and study the Dirac operator on $\partial \Omega \times(-\epsilon, \epsilon)$. Let $e_{1}, e_{2}, e_{3}$ be defined as in Section 4.5, and let $\theta^{1}, \theta^{2}, \theta^{3}$ be the dual coframe. Then $\theta^{3}=d t$. Let

$$
\mathbf{D}^{t}=c\left(\theta^{3}\right)\left(c\left(\theta^{1}\right) \nabla_{1}^{t}+c\left(\theta^{2}\right) \nabla_{2}^{t}\right)
$$

where $\nabla^{t}$ is the Levi-Civita connection on $\Sigma_{t}$. Then

$$
\mathbf{D} \psi=c\left(\theta^{3}\right)\left(\frac{\partial \psi}{\partial t}-\mathbf{D}^{t} \psi+\frac{H}{2} \psi\right) .
$$

Let

$$
\mathbf{D}^{\prime}=\mathbf{D}-\frac{1}{2} \beta(t) H c\left(\theta^{3}\right),
$$

where $\beta:(-\epsilon, \epsilon) \rightarrow \mathbb{R}$ is a smooth function such that $\beta(t)=\beta(-t)$ and

$$
\beta(t)= \begin{cases}1 & |t| \leq \epsilon / 3 \\ 0 & |t|>2 \epsilon / 3\end{cases}
$$

Then $\mathbf{D}^{\prime}$ extends to a first-order differential operator on $M$ with smooth coefficients.

In Section 4.9, we will prove the following existence and uniqueness of the Dirac spinor with prescribed asymptotics.

Theorem 8. Let $\psi^{1}, \ldots, \psi^{\ell}$ be constant spinors defined on the ends $E^{1}, \ldots, E^{\ell}$. Then there exists a unique spinor $\psi \in W_{\text {loc }}^{1,2}(M, S)$ such that

(1) $\mathbf{D} \psi=0$;

(2) $\psi \in C^{\infty}(M \backslash \partial \Omega, S)$;

(3) $\psi \in W_{\text {loc }}^{1, p}(M, S)$ for any $2 \leq p<\infty$.

(4) On each end $E^{\alpha}$, let $\rho$ be defined as in Theorem 3 , Then

$$
\lim _{\rho \rightarrow \infty} \rho^{1-\epsilon}\left|\psi-\psi^{\alpha}\right|=0
$$

for any $\epsilon>0$.

In general, the mean curvature along $\partial \Omega$ is discontinuous, so the Dirac spinor $\psi$ in Theorem 8 is not in $C^{1}(M, S)$. 
4.7. Boundary values of $W^{1,2}$ functions. We recall the following result from [25], where $H_{p}^{m}$ corresponds to $W^{m, p}$ in our notation.

Theorem 9 ([25, Theorem 3.4.5]). If $G$ is bounded and of class $C_{1}^{m-1}$ the functions $u \in C_{p}^{m-1}(\bar{G})$ are dense in any space $H_{p}^{m}(G)$ with $p \geq 1$ and there is a bounded operator $B$ from $H_{p}^{m}(G)$ into $H_{p}^{m-1}(\partial G)$ such that $B u=\left.u\right|_{\partial G}$ whenever $u \in C_{1}^{m-1}(\bar{G})$. If $u_{n} \rightarrow u$ in $H_{p}^{m}(G)$, then $u_{n} \rightarrow u$ in $H_{p}^{m-1}(\partial G)$. If $p>1$, the mapping is compact.

Recall that we have translated $\Sigma_{0}^{\alpha} \subset \mathbb{R}^{3}$ such that there is an $a>0$ such that the closed ball $B_{a}$ of radius $a$ centered at the origin is disjoint from $E^{\alpha}$. Choose $L>0$ such that $E^{\alpha}$ contains $\mathbb{R}^{3} \backslash B_{L}$. For $r>L$, let

$$
E_{r}^{\alpha}=E^{\alpha} \backslash B_{r}, \quad S_{r}^{\alpha}=\partial E_{r}^{\alpha}, \quad M_{r}=M \backslash \bigcup_{\alpha=1}^{\ell} E_{r}^{\alpha} .
$$

For a fixed $r>L$, let $G_{+}$be the interior of $\Omega$, and let $G_{-}$be the interior of $M_{r} \backslash \Omega$. We have a disjoint union

$$
M_{r}=G_{+} \cup \partial \Omega \cup G_{-} \cup \partial M_{r},
$$

where

$$
\bar{G}_{+}=G_{+} \cup \partial \Omega, \quad \bar{G}_{-}=\partial \Omega \cup G_{-} \cup \partial M_{r} .
$$

Let $r_{ \pm}: W^{1,2}(M) \rightarrow W^{1,2}\left(G_{ \pm}\right)$be the restriction map, and let $b_{ \pm}: W^{1,2}\left(G_{ \pm}\right) \rightarrow$ $L^{2}(\partial \Omega)$ be the bounded linear operator in Theorem 9. Let $B_{ \pm}=b_{ \pm} \circ r_{ \pm}$: $W^{1,2}(M) \rightarrow L^{2}(\partial \Omega)$. Given $u \in W^{1,2}(M)$, there exists a sequence $\left\{u_{n}\right\} \subset$ $C_{1}^{m-1}\left(M_{r}\right)$ such that $\left.u_{n} \rightarrow u\right|_{\Omega \cup G_{-}}$in $W^{1,2}\left(\Omega \cup G_{-}\right)$. Then $B_{+} u=B_{-} u=$ $\lim _{n \rightarrow \infty}\left(\left.u_{n}\right|_{\partial \Omega}\right)$.

4.8. Estimates near $\Omega$. Let $L>0$ be chosen as in Section 4.7. For $r>L$, let $M_{r}, S_{r}^{\alpha}$ be defined as in Section 4.7 The goal of this subsection is to establish the following estimate, which will be a crucial ingredient in the proof of Theorem 8

Proposition 10. For $r>L$ and $\psi \in W_{\text {loc }}^{1,2}(M, S) \cap C^{\infty}\left(M \backslash M_{L}, S\right)$, we have

$$
2 \int_{M_{r}}|\mathbf{D} \psi|^{2} \geq \frac{1}{10} \int_{M_{r}}|\nabla \psi|^{2}+\frac{1}{16} \int_{\Omega} u^{-2}|d u|^{2}|\psi|^{2}+\sum_{\alpha=1}^{\ell} \int_{S_{r}^{\alpha}}\left\langle\frac{H}{2} \psi-c(\nu) \check{\mathbf{D}} \psi, \psi\right\rangle
$$

where $\check{\mathbf{D}}$ is the Dirac operator on $S_{r}^{\alpha}$.

Lemma 11. Let $U$ be an open set of $M$. For any spinor $\eta \in W_{0}^{1,2}(U, S), \psi \in$ $W_{\mathrm{loc}}^{1,2}(U, S)$, we have

$$
\begin{gathered}
\int_{U}\langle\mathbf{D} \psi, \eta\rangle=\int_{U}\langle\psi, \mathbf{D} \eta\rangle \\
\int_{U}\langle\mathbf{D} \psi, \mathbf{D} \eta\rangle=\int_{U}\langle\nabla \psi, \nabla \eta\rangle_{\tilde{g}}+\int_{\partial \Omega \cap U}\left(2 \bar{\nu}(u)+\frac{1}{2}\langle X, \bar{\nu}\rangle\right)\langle\psi, \eta\rangle .
\end{gathered}
$$

Proof. By the discussion in Section 4.7, the right-hand side of (29) makes sense. It suffices to show that (28) and (29) hold for $\psi \in C^{\infty}(U, S), \eta \in C_{0}^{\infty}(U, S)$. 
Let $U_{1}=\Omega \cap U, U_{2}=U \backslash \bar{\Omega}$, and $I=\partial \Omega \cap U$. We have

$$
\begin{aligned}
\int_{U_{1}}\langle\mathbf{D} \psi, \eta\rangle & =\int_{U_{1}}\langle\psi, \mathbf{D} \eta\rangle+\int_{I}\langle c(\hat{\nu}) \psi, \eta\rangle, \\
\int_{U_{2}}\langle\mathbf{D} \psi, \eta\rangle & =\int_{U_{2}}\langle\psi, \mathbf{D} \eta\rangle+\int_{I}\langle-c(\hat{\nu}) \psi, \eta\rangle,
\end{aligned}
$$

where $\hat{\nu}$ is the outward unit normal of $\partial \Omega$ in $(\Omega, \hat{g})$ (see e.g. [6, Proposition 3.4]). Equation (28) is the sum of (30) and (31).

We also have

$$
\begin{aligned}
\int_{U_{1}}\langle\mathbf{D} \psi, \mathbf{D} \eta\rangle & =\int_{U_{1}}\langle\nabla \psi, \nabla \eta\rangle_{\tilde{g}}+\int_{I}\left\langle\frac{1}{2}(\bar{H}+4 \bar{\nu}(u)) \psi-c(\hat{\nu}) \check{\mathbf{D}} \psi, \eta\right\rangle \\
\int_{U_{2}}\langle\mathbf{D} \psi, \mathbf{D} \eta\rangle & =\int_{U_{2}}\langle\nabla \psi, \nabla \eta\rangle_{\tilde{g}}+\int_{I}\left\langle\frac{1}{2}(\langle X, \bar{\nu}\rangle-\bar{H}) \psi+c(\hat{\nu}) \check{\mathbf{D}} \psi, \eta\right\rangle
\end{aligned}
$$

where $\check{\mathbf{D}}$ is the Dirac operator on $\partial \Omega$ (see e.g. [18]). Equation (29) is the sum of (32) and (33).

The proof of Lemma 11 also gives the following:

Lemma 12. For $r>L$ and $\psi, \eta \in W_{\mathrm{loc}}^{1,2}(M, S)$, we have

$$
\int_{M_{r}}\langle\mathbf{D} \psi, \eta\rangle=\int_{M_{r}}\langle\psi, \mathbf{D} \eta\rangle+\sum_{\alpha=1}^{\ell} \int_{S_{r}^{\alpha}}\langle c(\nu) \psi, \eta\rangle .
$$

For $r>L, \psi \in W_{\mathrm{loc}}^{1,2}(M, S) \cap C^{\infty}\left(M \backslash M_{L}, S\right)$, and $\eta \in W_{\mathrm{loc}}^{1,2}(M, S)$, we have

$$
\begin{aligned}
\int_{M_{r}}\langle\mathbf{D} \psi, \mathbf{D} \eta\rangle= & \int_{M_{r}}\langle\nabla \psi, \nabla \eta\rangle_{\tilde{g}}+\int_{\partial \Omega}\left(2 \bar{\nu}(u)+\frac{1}{2}\langle X, \bar{\nu}\rangle\right)\langle\psi, \eta\rangle \\
& +\sum_{\alpha=1}^{\ell} \int_{S_{r}^{\alpha}}\left\langle\frac{H}{2} \psi-c(\nu) \check{\mathbf{D}} \psi, \eta\right\rangle .
\end{aligned}
$$

Lemma 13. Let $\overline{\mathbf{D}}$ and $\mathbf{D}$ denote the Dirac operators on $\left.S\right|_{\Omega}$ defined by the LeviCivita connections of $\bar{g}$ and $\hat{g}=u^{4} \bar{g}$, respectively. Then

$$
\mathbf{D} \psi=\frac{1}{u^{2}} \overline{\mathbf{D}} \psi+\frac{1}{2 u^{3}} \bar{c}(d u) \psi
$$

where $\bar{c}$ is the Clifford multiplication defined by $\bar{g}$.

Proof. The tangent bundle of $\Omega$ is trivial, so there exists a global orthonormal frame $\left\{\bar{e}_{1}, \bar{e}_{2}, \bar{e}_{3}\right\}$ with respect to $\bar{g}$. Let $\left\{\bar{\theta}^{1}, \bar{\theta}^{2}, \bar{\theta}^{2}\right\}$ be the dual coframe. We have

$$
\begin{aligned}
\bar{\nabla}_{\bar{e}_{i}} \bar{e}_{j} & =\bar{\Gamma}_{i j}^{k} \bar{e}_{k}, \\
d \bar{\theta}^{i} & =-\bar{\Gamma}_{k j}^{i} \bar{\theta}^{k} \wedge \bar{\theta}^{j} \\
\bar{\nabla}_{\bar{e}_{i}} \psi & =\bar{e}_{i}(\psi)+\frac{1}{4} \bar{\Gamma}_{i j}^{k} \bar{c}\left(\bar{\theta}^{j}\right) \bar{c}\left(\bar{\theta}^{k}\right) \psi .
\end{aligned}
$$

Let

$$
e_{i}=u^{-2} \bar{e}_{i}, \quad \theta^{i}=u^{2} \bar{\theta}^{i} .
$$


Then $\left\{e_{1}, e_{2}, e_{3}\right\}$ is a global orthonormal frame with respect to $\hat{g}$, and $\left\{\theta^{1}, \theta^{2}, \theta^{3}\right\}$ is the coframe. Let $u_{i}=\bar{e}_{i}(u), \psi_{i}=\bar{\nabla}_{\bar{e}_{i}} \psi$. Then

$$
\begin{aligned}
d \theta^{i} & =2 u_{j} u \bar{\theta}^{j} \wedge \bar{\theta}^{i}-\sum_{j, k} u^{2} \bar{\Gamma}_{k j}^{i} \bar{\theta}^{k} \wedge \bar{\theta}^{j}=-\left(\bar{\Gamma}_{k j}^{i} \bar{\theta}^{k}+\frac{2 u_{j}}{u} \bar{\theta}^{i}\right) \wedge \theta^{j}, \\
\Gamma_{k j}^{i} & =\left(\bar{\Gamma}_{l j}^{i} \bar{\theta}^{l}+\frac{2 u_{j}}{u} \bar{\theta}^{i}\right)\left(e_{k}\right)=u^{-2} \bar{\Gamma}_{k j}^{i}+2 u^{-3} u_{j} \delta_{i k}, \\
\nabla_{e_{i}} \psi & =e_{i}(\psi)+\frac{1}{4} \Gamma_{i j}^{k} c\left(\theta^{j}\right) c\left(\theta^{k}\right) \psi=\frac{1}{u^{2}} \psi_{i}+\frac{1}{2 u^{3}} \bar{c}(d u) \bar{c}\left(\bar{\theta}^{i}\right) \psi .
\end{aligned}
$$

Note that $\bar{c}\left(\bar{\theta}^{i}\right)=c\left(\theta^{i}\right)$, so

$$
\mathbf{D} \psi=c\left(\theta^{i}\right) \nabla_{e_{i}} \psi=\frac{1}{u^{2}} \bar{c}\left(\bar{\theta}^{i}\right) \psi_{i}+\frac{1}{2 u^{3}} \bar{c}\left(\bar{\theta}^{i}\right) \bar{c}(d u) \bar{c}\left(\bar{\theta}^{i}\right) \psi=\frac{1}{u^{2}} \overline{\mathbf{D}} \psi+\frac{1}{2 u^{3}} \bar{c}(d u) \psi .
$$

\section{Lemma 14.}

$$
|\bar{\nabla} \psi|_{\bar{g}}^{2}=u^{4}|\nabla \psi|_{\hat{g}}^{2}+\frac{3 u^{2}}{4}|d u|_{\hat{g}}^{2}|\psi|^{2}-u^{3} \operatorname{Re}\langle\mathbf{D} \psi, c(d u) \psi\rangle+2 u^{3} \operatorname{Re}\left\langle e_{i}(u) \nabla_{e_{i}} \psi, \psi\right\rangle .
$$

Proof. We use the notation in the proof of Lemma 13, where we calculated that

$$
\nabla_{e_{i}} \psi=\frac{1}{u^{2}} \psi_{i}+\frac{1}{2 u^{3}} \bar{c}(d u) \bar{c}\left(\bar{\theta}^{i}\right) \psi
$$

Note that $\left\langle\psi_{1}, \psi_{2}\right\rangle$ does not depend on the metric on the tangent bundle of $\Omega$. We have

$$
\left|\nabla_{e_{i}} \psi\right|^{2}=\frac{1}{u^{4}}\left|\psi_{i}\right|^{2}+\frac{|d u|_{\bar{g}}^{2}}{4 u^{4}}|\psi|^{2}+\frac{1}{u^{5}} \operatorname{Re}\left\langle\psi_{i}, \bar{c}(d u) \bar{c}\left(\bar{\theta}^{i}\right) \psi\right\rangle .
$$

Note that the last term on the right-hand side of (40) is not a sum and can be rewritten as follows:

$$
\begin{aligned}
\left\langle\psi_{i}, \bar{c}(d u) \bar{c}\left(\bar{\theta}^{i}\right) \psi\right\rangle & =\left\langle\psi_{i}, u_{j} \bar{c}\left(\bar{\theta}^{j}\right) \bar{c}\left(\bar{\theta}^{i}\right) \psi\right\rangle \\
& =\left\langle\psi_{i}, u_{j}\left(-\bar{c}\left(\bar{\theta}^{i}\right) \bar{c}\left(\bar{\theta}^{j}\right)-2 \delta_{i j}\right) \psi\right\rangle \\
& =\left\langle\bar{c}\left(\bar{\theta}^{i}\right) \psi_{i}, u_{j} \bar{c}\left(\bar{\theta}^{j}\right) \psi\right\rangle-2 u_{i}\left\langle\psi_{i}, \psi\right\rangle .
\end{aligned}
$$

We now sum over $i=1,2,3$ and obtain

$$
\begin{aligned}
|\nabla \psi|_{\hat{g}}^{2} & =\sum_{i=1}^{3}\left|\nabla_{e_{i}} \psi\right|^{2} \\
& =\frac{1}{u^{4}}|\bar{\nabla} \psi|_{\bar{g}}^{2}+\frac{3|d u|_{\bar{g}}^{2}}{4 u^{6}}|\psi|^{2}+\frac{1}{u^{5}} \operatorname{Re}\left(\langle\overline{\mathbf{D}} \psi, \bar{c}(d u) \psi\rangle-2 u_{i}\left\langle\psi_{i}, \psi\right\rangle\right) .
\end{aligned}
$$

This proves (37). By symmetry, we have

$$
\begin{gathered}
|\bar{\nabla} \psi|_{\bar{g}}^{2}=u^{4}|\nabla \psi|_{\hat{g}}^{2}+\frac{3 u^{6}}{4}\left|d\left(u^{-1}\right)\right|_{g}^{2}|\psi|^{2}+u^{5} \operatorname{Re}\left\langle\mathbf{D} \psi, c\left(d\left(u^{-1}\right)\right) \psi\right\rangle \\
-2 u^{5} \operatorname{Re}\left\langle e_{i}\left(u^{-1}\right) \nabla_{e_{i}} \psi, \psi\right\rangle
\end{gathered}
$$

which is equivalent to (38). 
Lemma 15. Let $d \bar{\sigma}$ denote the volume form of $\bar{g}$, and let div denote the divergence defined by $\bar{g}$. Then

$$
\int_{\Omega}|\nabla \psi|_{\hat{g}}^{2} d \sigma=\int_{\Omega}\left(u^{2}|\bar{\nabla} \psi|_{\bar{g}}^{2}+\frac{3}{4}|d u|_{\bar{g}}^{2}|\psi|^{2}+u \operatorname{Re}\langle\overline{\mathbf{D}} \psi, \bar{c}(d u) \psi\rangle-2 u \operatorname{Re}\left\langle u_{i} \psi_{i}, \psi\right\rangle\right) d \bar{\sigma},
$$

$$
\begin{aligned}
2 \int_{\partial \Omega} \bar{\nu}(u)|\psi|^{2} \geq \int_{\Omega}(2 \mid & \left.d u\right|_{\bar{g}} ^{2}|\psi|^{2}+\frac{1}{2}|X|_{\bar{g}}^{2} u^{2}|\psi|^{2} \\
& \left.-\frac{1}{2}(\operatorname{div} X) u^{2}|\psi|^{2}+4 u \operatorname{Re}\left\langle u_{i} \psi_{i}, \psi\right\rangle\right) d \bar{\sigma}
\end{aligned}
$$

$$
\frac{1}{2} \int_{\partial \Omega}\langle X, \bar{\nu}\rangle|\psi|^{2} \geq \int_{\Omega}\left(\frac{1}{2}(\operatorname{div} X) u^{2}|\psi|^{2}-\frac{1}{2}|X|_{\bar{g}}^{2} u^{2}|\psi|^{2}-\frac{\left|\bar{\nabla}\left(u^{2}|\psi|^{2}\right)\right|_{\bar{g}}^{2}}{8 u^{2}|\psi|^{2}}\right) d \bar{\sigma} .
$$

Proof. We have $d \bar{\sigma}=u^{6} d \sigma$, so (41) follows from (37). To prove (42), note that

$$
\begin{aligned}
2 \int_{\partial \Omega} \bar{\nu}(u)|\psi|^{2} d \sigma= & 2 \int_{\Omega} \operatorname{div}\left(u \bar{\nabla} u|\psi|^{2}\right) d \bar{\sigma} \\
= & 2 \int_{\Omega}|\bar{\nabla} u|_{\bar{g}}^{2}|\psi|^{2} d \bar{\sigma}+2 \int_{\Omega} u \bar{\Delta} u|\psi|^{2} d \bar{\sigma} \\
& +2 \int_{\Omega} u\left\langle\bar{\nabla} u, \bar{\nabla}\left(|\psi|^{2}\right)\right\rangle_{\bar{g}} d \bar{\sigma}
\end{aligned}
$$

where

$$
\begin{gathered}
\bar{\Delta} u=\frac{\bar{R}}{8} u \geq \frac{1}{4}\left(|X|_{\bar{g}}^{2}-\operatorname{div} X\right) u, \\
\left\langle\bar{\nabla} u, \bar{\nabla}\left(|\psi|^{2}\right)\right\rangle_{\bar{g}}=u_{i} \bar{e}_{i}\left(|\psi|^{2}\right)=2 \operatorname{Re}\left\langle u_{i} \psi_{i}, \psi\right\rangle,
\end{gathered}
$$

so (42) holds. Finally,

$$
\begin{aligned}
\frac{1}{2} \int_{\partial \Omega}\langle X, \bar{\nu}\rangle|\psi|^{2} & =\frac{1}{2} \int_{\Omega} \operatorname{div}\left(X u^{2}|\psi|^{2}\right) d \bar{\sigma} \\
& =\frac{1}{2} \int_{\Omega}(\operatorname{div} X) u^{2}|\psi|^{2} d \bar{\sigma}+\frac{1}{2} \int_{\Omega}\left\langle X, \bar{\nabla}\left(u^{2}|\psi|^{2}\right)\right\rangle_{\bar{g}} d \bar{\sigma} \\
& =\frac{1}{2} \int_{\Omega}(\operatorname{div} X) u^{2}|\psi|^{2} d \bar{\sigma}+2 \int_{\Omega}\left\langle\frac{1}{\sqrt{2}} X u|\psi|, \frac{\bar{\nabla}\left(u^{2}|\psi|^{2}\right.}{\sqrt{8} u|\psi|}\right\rangle_{\bar{g}} d \bar{\sigma} \\
& \geq \frac{1}{2} \int_{\Omega}(\operatorname{div} X) u^{2}|\psi|^{2} d \bar{\sigma}-\frac{1}{2} \int_{\Omega}|X|_{\bar{g}}^{2} u^{2}|\psi|^{2} d \bar{\sigma} \\
& -\frac{1}{8} \int_{\Omega} \frac{\left|\bar{\nabla}\left(u^{2}|\psi|^{2}\right)\right|_{\bar{g}}^{2}}{u^{2}|\psi|^{2}} d \bar{\sigma}
\end{aligned}
$$

This proves (43).

\section{Lemma 16.}

$$
\begin{aligned}
\int_{\Omega}|\nabla \psi|_{\hat{g}}^{2} d \sigma+2 \int_{\partial \Omega} & \bar{\nu}(u)|\psi|^{2}+\frac{1}{2} \int_{\partial \Omega}\langle X, \bar{\nu}\rangle|\psi|^{2} \\
& \geq \int_{\Omega}\left(\frac{1}{10}|\nabla \psi|_{\hat{g}}^{2}+\frac{1}{16 u^{2}}|d u|_{\hat{g}}^{2}|\psi|^{2}-|\mathbf{D} \psi|^{2}\right) d \sigma .
\end{aligned}
$$


Proof. By Lemma 15, we have

$$
\begin{gathered}
\int_{\Omega}|\nabla \psi|_{\hat{g}}^{2} d \sigma+2 \int_{\partial \Omega} \bar{\nu}(u)|\psi|^{2}+\frac{1}{2} \int_{\partial \Omega}\langle X, \bar{\nu}\rangle|\psi|^{2} \\
\geq \int_{\Omega}\left(u^{2}|\bar{\nabla} \psi|_{\bar{g}}^{2}+\frac{11}{4}|d u|_{\bar{g}}^{2}|\psi|^{2}+2 u \operatorname{Re}\left\langle u_{i} \psi_{i}, \psi\right\rangle\right. \\
\left.-\frac{1}{8} \frac{\left|\bar{\nabla}\left(u^{2}|\psi|^{2}\right)\right|^{2}}{u^{2}|\psi|^{2}}+u \operatorname{Re}\langle\mathbf{D} \psi, \bar{c}(d u) \psi\rangle\right) d \bar{\sigma} .
\end{gathered}
$$

We rewrite

$$
-\frac{1}{8} \frac{\left|\bar{\nabla}\left(u^{2}|\psi|^{2}\right)\right|^{2}}{u^{2}|\psi|^{2}}
$$

as follows:

$$
\begin{aligned}
\bar{\nabla}\left(u^{2}|\psi|^{2}\right) & =2 u \bar{\nabla} u|\psi|^{2}+u^{2} 2 \operatorname{Re}\langle\bar{\nabla} \psi, \psi\rangle, \\
\left|\bar{\nabla}\left(u^{2}|\psi|^{2}\right)\right|^{2} & =4 u^{2}|d u|_{\bar{g}}^{2}|\psi|^{4}+4 u^{4} \sum_{i=1}^{3}\left(\operatorname{Re}\left\langle\psi_{i}, \psi\right\rangle\right)^{2}+8 u^{3}|\psi|^{2} \operatorname{Re}\left\langle u_{i} \psi_{i}, \psi\right\rangle, \\
-\frac{\left|\bar{\nabla}\left(u^{2}|\psi|^{2}\right)\right|^{2}}{8 u^{2}|\psi|^{2}} & =-\frac{1}{2}|d u|_{\bar{g}}^{2}|\psi|^{2}-\frac{1}{2} \frac{u^{2}}{|\psi|^{2}} \sum_{i=1}^{3}\left(\operatorname{Re}\left\langle\psi_{i}, \psi\right\rangle\right)^{2}-u \operatorname{Re}\left\langle u_{i} \psi_{i}, \psi\right\rangle \\
& \geq-\frac{1}{2}|d u|_{\bar{g}}^{2}|\psi|^{2}-\frac{1}{2} u^{2}|\bar{\nabla} \psi|_{\bar{g}}^{2}-u \operatorname{Re}\left\langle u_{i} \psi_{i}, \psi\right\rangle .
\end{aligned}
$$

So

$$
\begin{gathered}
\int_{\Omega}|\nabla \psi|_{\hat{g}}^{2} d \sigma+2 \int_{\partial \Omega} \bar{\nu}(u)|\psi|^{2}+\frac{1}{2} \int_{\partial \Omega}\langle X, \bar{\nu}\rangle|\psi|^{2} \\
\geq \int_{\Omega}\left(\frac{1}{2} u^{2}|\bar{\nabla} \psi|_{\bar{g}}^{2}+\frac{9}{4}|d u|_{\bar{g}}^{2}|\psi|^{2}+u \operatorname{Re}\left\langle u_{i} \psi_{i}, \psi\right\rangle+u \operatorname{Re}\langle\overline{\mathbf{D}} \psi, \bar{c}(d u) \psi\rangle\right) d \bar{\sigma} .
\end{gathered}
$$

In the rest of this proof, we will write

$$
|d u|^{2}=|d u|_{\hat{g}}^{2}, \quad|\nabla \psi|^{2}=|\nabla \psi|_{\hat{g}}^{2}
$$

By (38),

$$
\frac{1}{2} u^{2}|\bar{\nabla} \psi|_{\bar{g}}^{2}=\frac{u^{6}}{2}|\nabla \psi|^{2}+\frac{3 u^{4}}{8}|d u|^{2}|\psi|^{2}-\frac{u^{5}}{2} \operatorname{Re}\langle\mathbf{D} \psi, c(d u) \psi\rangle+u^{5} \operatorname{Re}\left\langle e_{i}(u) \nabla_{e_{i}} \psi, \psi\right\rangle .
$$

By (39) and symmetry,

$$
\psi_{i}=u^{2} \nabla_{e_{i}} \psi+\frac{u^{3}}{2} c\left(d\left(u^{-1}\right)\right) c\left(\theta^{i}\right) \psi=u^{2} \nabla_{e_{i}} \psi-\frac{u}{2} c(d u) c\left(\theta^{i}\right) \psi
$$

So

$$
\begin{aligned}
u \operatorname{Re}\left\langle u_{i} \psi_{i}, \psi\right\rangle & =u \operatorname{Re}\left\langle u^{2} e_{i}(u)\left(u^{2} \nabla_{e_{i}} \psi-\frac{u}{2} c(d u) c\left(\theta^{i}\right)\right) \psi, \psi\right\rangle \\
& =u^{5} \operatorname{Re}\left\langle e_{i}(u) \nabla_{e_{i}} \psi, \psi\right\rangle-\frac{u^{4}}{2} \operatorname{Re}\left\langle c(d u)^{2} \psi, \psi\right\rangle,
\end{aligned}
$$

which implies

$$
u \operatorname{Re}\left\langle u_{i} \psi_{i}, \psi\right\rangle=u^{5} \operatorname{Re}\left\langle e_{i}(u) \nabla_{e_{i}} \psi, \psi\right\rangle+\frac{u^{4}}{2}|d u|^{2}|\psi|^{2} .
$$


We also have

$$
\begin{aligned}
u \operatorname{Re}\langle\overline{\mathbf{D}} \psi, \bar{c}(d u) \psi\rangle & =u \operatorname{Re}\left\langle u^{2} \mathbf{D} \psi-\frac{1}{2 u} \bar{c}(d u) \psi, \bar{c}(d u) \psi\right\rangle \\
& =u^{5} \operatorname{Re}\langle\mathbf{D} \psi, c(d u) \psi\rangle-\frac{1}{2} u^{4}\langle c(d u) \psi, c(d u) \psi\rangle
\end{aligned}
$$

which implies

$$
u \operatorname{Re}\langle\overline{\mathbf{D}} \psi, \bar{c}(d u) \psi\rangle=u^{5} \operatorname{Re}\langle\mathbf{D} \psi, c(d u) \psi\rangle-\frac{1}{2} u^{4}|d u|^{2}|\psi|^{2} .
$$

Let $v=\log (u)$. Then (44), (45), (47), and (48) imply that

$$
\begin{aligned}
& \int_{\Omega}|\nabla \psi|_{\hat{g}}^{2} d \sigma+2 \int_{\partial \Omega} \bar{\nu}(u)|\psi|^{2}+\frac{1}{2} \int_{\partial \Omega}\langle X, \bar{\nu}\rangle|\psi|^{2} \\
\geq & \int_{\Omega}\left(\frac{1}{2}|\nabla \psi|^{2}+\frac{21}{8}|d v|^{2}|\psi|^{2}+\frac{1}{2} \operatorname{Re}\langle\mathbf{D} \psi, c(d v) \psi\rangle+2 \operatorname{Re}\left\langle e_{i}(v) \nabla_{e_{i}} \psi, \psi\right\rangle\right) d \sigma \\
\geq & \int_{\Omega}\left(\frac{1}{2}|\nabla \psi|^{2}+\frac{21}{8}|d v|^{2}|\psi|^{2}-\left(|\mathbf{D} \psi|^{2}+\frac{1}{16}|d v|^{2}|\psi|^{2}\right)\right. \\
\left.-\left(\frac{2}{5}|\nabla \psi|^{2}+\frac{5}{2}|d v|^{2}|\psi|^{2}\right)\right) d \sigma & \int_{\Omega}\left(\frac{1}{10}|\nabla \psi|^{2}+\frac{1}{16}|d v|^{2}|\psi|^{2}-|\mathbf{D} \psi|^{2}\right) d \sigma
\end{aligned}
$$

Proof of Proposition 10. By (35),

$$
\begin{aligned}
\int_{M_{r}}|\mathbf{D} \psi|^{2}= & \int_{M_{r} \backslash \Omega}|\nabla \psi|^{2}+\int_{\Omega}|\nabla \psi|^{2}+\int_{\partial \Omega}\left(2 \bar{\nu}(u)+\frac{1}{2}\langle X, \bar{\nu}\rangle\right)|\psi|^{2} \\
& +\sum_{\alpha=1}^{\ell} \int_{S_{r}^{\alpha}}\left\langle\frac{H}{2} \psi-c(\nu) \check{\mathbf{D}} \psi, \psi\right\rangle,
\end{aligned}
$$

where

$$
\int_{\Omega}|\nabla \psi|^{2}+\int_{\partial \Omega}\left(2 \bar{\nu}(u)+\frac{1}{2}\langle X, \bar{\nu}\rangle\right)|\psi|^{2} \geq \frac{1}{10} \int_{\Omega}|\nabla \psi|^{2}+\frac{1}{16} \int_{\Omega} u^{-2}|d u|^{2}|\psi|^{2}-\int_{\Omega}|\mathbf{D} \psi|^{2}
$$

by Lemma 16. Therefore,

$$
\begin{aligned}
& \int_{M_{r}}|\mathbf{D} \psi|^{2}+\int_{\Omega}|\mathbf{D} \psi|^{2} \\
\geq & \int_{M_{r} \backslash \Omega}|\nabla \psi|^{2}+\frac{1}{10} \int_{\Omega}|\nabla \psi|^{2}+\frac{1}{16} \int_{\Omega} u^{-2}|d u|^{2}|\psi|^{2} \\
& +\sum_{\alpha=1}^{\ell} \int_{S_{r}^{\alpha}}\left\langle\frac{H}{2} \psi-c(\nu) \check{\mathbf{D}} \psi, \psi\right\rangle,
\end{aligned}
$$

which implies (27). 
4.9. Proof of Theorem 8 . We modify Parker and Taubes's proof of [27, Theorem 4.1]. In [27, the hypersurface Dirac operator is studied, so the second fundamental form of the spacelike hypersurface in the spacetime is involved in estimates. Here we consider the Riemannian case, so the estimates are simpler in certain steps. The main difficulty in our case comes from the discontinuity of the zeroth-order term of the Dirac operator along $\partial \Omega$.

Let $L>0$ be chosen as in Section 4.7. For $r>L$, let $E_{r}^{\alpha}, M_{r}, S_{r}^{\alpha}$ be defined as in Section 4.7. Choose a smooth function $\beta_{L}^{\alpha}$ on each end $E^{\alpha}$ such that (i) $0 \leq \beta_{L}^{\alpha} \leq 1$, (ii) $\beta_{L}^{\alpha} \equiv 1$ on $E_{3 L}^{\alpha}$, (iii) $\beta_{L}^{\alpha} \equiv 0$ on $E^{\alpha} \cap M_{2 L}$, and (iv) $\left|\nabla \beta_{L}^{\alpha}\right| \leq 2 / L$. Then $\beta_{L}^{\alpha} \psi^{\alpha}$ extends to a smooth section of $S$ over $M$. Define

$$
\psi_{0}=\sum_{\alpha=1}^{\ell} \beta_{L}^{\alpha} \psi^{\alpha} \in C^{\infty}(M, S) .
$$

We wish to find $\psi_{1} \in W^{1,2}(M, S)$ such that

$$
\mathbf{D} \psi_{1}=-\mathbf{D} \psi_{0}
$$

and

$$
\lim _{r \rightarrow \infty} r^{1-\epsilon}\left|\psi_{1}\right|=0
$$

Then $\psi=\psi_{0}+\psi_{1}$ is the desired solution.

Let $U$ be an open subset of $M$, and let $\psi \in L_{\text {loc }}^{2}(U, S)$. Given $\eta \in C^{\infty}(U, S), \psi$ is said to satisfy

$$
\mathbf{D} \psi=\eta
$$

in the weak sense if

$$
\int_{U}\langle\psi, \mathbf{D} \phi\rangle=\int_{U}\langle\eta, \phi\rangle
$$

for any $\phi \in C_{0}^{1}(U, S)$, or equivalently, for any $\phi \in W_{\text {loc }}^{1,2}(U, S)$.

Lemma 17. Let $U$ be an open subset of $M$, and let $\psi \in L_{\mathrm{loc}}^{2}(U, S)$ be a weak solution to

$$
\mathbf{D} \psi=\eta,
$$

where $\eta \in C^{\infty}(U, S)$. Then $\psi \in C^{\infty}(U \backslash \partial \Omega, S)$, and $\psi \in W_{\text {loc }}^{1, p}(U, S)$ for any $2 \leq p<\infty$.

Proof. Recall from Section 4.6 that $\mathbf{D}=\mathbf{D}^{\prime}+A$, where $\mathbf{D}^{\prime}$ is a first-order elliptic operator with smooth coefficients, and $A \in L^{\infty}(U, \operatorname{End}(S)) \cap C^{\infty}(U \backslash \partial \Omega$, End $(S))$. So $\psi \in C^{\infty}(U \backslash \partial \Omega, S)$ by elliptic regularity. Now

$$
\mathbf{D}^{\prime} \psi=A \psi+\eta,
$$

where $A \psi+\eta \in L_{\text {loc }}^{2}(U, S)$. By elliptic regularity, $\psi \in W_{\text {loc }}^{1,2}(U) \subset L_{\text {loc }}^{6}(U)$, so $A \psi+\eta \in L_{\text {loc }}^{6}(U)$. By elliptic regularity again, $\psi \in W_{\text {loc }}^{1,6}(U, S) \subset C^{0}(U, S)$. So $A \psi+\eta \in L_{\mathrm{loc}}^{p}(U, S)$ for any $p \geq 2$, which implies $\psi \in W_{\mathrm{loc}}^{1, p}(U, S)$ for any $p \geq 2$.

We recall some weighted Sobolev spaces introduced in [27. The distance function from the origin is a smooth function $\rho: E_{L}^{\alpha} \rightarrow \mathbb{R}$ such that $\rho\left(E_{L}^{\alpha}\right)=[L, \infty)$. This defined a smooth function $\rho$ on $M_{L}$ such that $\rho^{-1}(r)=\partial M_{r}$ for $r \geq L$. Fix a smooth function $\sigma$ on $M$ such that (i) $\sigma \geq 1$, (ii) $\sigma=\rho$ on $E_{2 L}^{\alpha}$ and (iii) $\sigma=1$ in $M_{L}$. 
Definition 18. Given a pair $(\delta, p)$ such that

$$
p \geq 2, \quad \frac{1}{2}-\frac{3}{p} \leq \delta \leq 2-\frac{3}{p}
$$

let $W_{\delta}^{0, p}, W_{\delta}^{1, p}$ be the completions of $C_{0}^{\infty}(M, S)$ with respect to the norms

$$
\|\psi\|_{0, \delta, p}=\left\|\sigma^{\delta} \psi\right\|_{p}, \quad\|\psi\|_{1, \delta, p}=\left\|\sigma^{1+\delta} \nabla \psi\right\|_{p}+\left\|\sigma^{\delta} \psi\right\|_{p},
$$

respectively, where $\|\psi\|_{p}$ is the $L^{p}$ norm.

Lemma 19. For $p \geq 2$ and $0<\delta<2-3 / p$, or $p=2$ and $\delta=-1$, the operators $\nabla$ and $\mathbf{D}$ are bounded linear maps from $W_{\delta}^{1, p}$ into $W_{\delta}^{0, p}$, and there is a continuous embedding $W_{\delta}^{1, p} \subset W=W_{-1}^{1,2}$.

Proof. It is obvious from the definitions that the operators $\nabla$ and $\mathbf{D}$ are bounded linear maps from $W_{\delta}^{1, p}$ into $W_{\delta+1}^{0, p}$. Suppose that $p \geq 2$ and $0<\delta<2-3 / p$. Then $\sigma^{-(1+\delta)}$ is in $L_{\frac{2 p}{p-2}}$. Set

$$
C=\left\|\sigma^{-(1+\delta)}\right\|_{\frac{2 p}{p-2}}
$$

which is a positive constant. We have

$$
\begin{aligned}
\|\psi\|_{1,-1,2} & =\|\nabla \psi\|_{2}+\left\|\sigma^{-1} \psi\right\|_{2} \\
& =\left\|\sigma^{-(1+\delta)} \sigma^{1+\delta} \nabla \psi\right\|_{2}+\left\|\sigma^{-(1+\delta)} \sigma^{\delta} \psi\right\|_{2} \\
& \leq\left\|\sigma^{-(1+\delta)}\right\|_{\frac{2 p}{p-2}}\|\nabla \psi\|_{p}+\left\|\sigma^{-(1+\delta)}\right\|_{\frac{2 p}{p-2}}\left\|\sigma^{\delta} \psi\right\|_{p} \\
& =C\|\psi\|_{1, \delta, p} .
\end{aligned}
$$

By Proposition 10,

$$
2 \int_{M_{r}}|\mathbf{D} \psi|^{2} \geq \frac{1}{10} \int_{M_{r}}|\nabla \psi|^{2}+\frac{1}{16} \int_{\Omega} u^{-2}|d u|^{2}|\psi|^{2}+\sum_{\alpha=1}^{\ell} \int_{S_{r}^{\alpha}}\left\langle\frac{H}{2} \psi-c(\nu) \check{\mathbf{D}} \psi, \psi\right\rangle
$$

for all $r \geq L$. So we have

$$
\int_{M}|\mathbf{D} \psi|^{2} \geq \frac{1}{20} \int_{M}|\nabla \psi|^{2}
$$

for $\psi \in C_{0}^{\infty}(N, S)$. By Lemma 19, (52) holds for $\psi \in W$, or equivalently,

Lemma 20. For $\psi \in W$, we have

$$
2 \sqrt{5}\|\mathbf{D} \psi\|_{2} \geq\|\nabla \psi\|_{2} .
$$

Corollary 21. For $p \geq 2$ and $0<\delta<2-3 / p$, or $p=2$ and $\delta=-1$, the operator $\mathbf{D}: W_{\delta}^{1, p} \rightarrow W_{\delta+1}^{0, p}$ is an injection.

Proof. Suppose that $\psi \in W_{\delta}^{1, p}$ and $\mathbf{D} \psi=0$. By Lemma 17, $\psi$ is continuous on $M$ and smooth on $M \backslash \partial \Omega$. By Lemma 20, $\nabla \psi=0$, so $|\psi|$ is constant outside $M \backslash \partial \Omega$. Then $|\psi|$ is constant on $M$ since $|\psi|$ is continuous on $M$. We have

$$
\|\psi\|_{1, \delta, p} \geq\left\|\sigma^{\delta} \psi\right\|_{p}=\left(\left\|\sigma^{\delta p}\right\|_{1}\right)^{1 / p}|\psi|,
$$

where $\delta p \geq-1$, so $\left\|\sigma^{\delta p}\right\|_{1}=\infty$. We must have $\psi \equiv 0$. 
Lemma 22 (27, Lemma 5.4 (a)]). For sufficiently large L, we have

$$
\left\|\sigma^{-1} \psi\right\|_{2 ; E_{2 L}^{\alpha}}^{2} \leq 5\|\nabla \psi\|_{2 ; E_{2 L}^{\alpha}}^{2}
$$

for $\psi \in W$.

Lemma 23. For sufficiently large $L$, there is a constant $c=c(L)>0$ such that for all $\psi \in W$,

$$
\|\psi\|_{W}^{2} \leq c\|\mathbf{D} \psi\|_{2}^{2} .
$$

Proof. We modify the proof of [27, Lemma 5.5].

Fix a large $L$ such that Lemma 22 holds. Choose a smooth function $\beta=\beta_{L}$ such that (i) $0 \leq \beta \leq 1$, (ii) $\beta \equiv 1$ on each $E_{3 L}^{\alpha}$, (iii) $\beta \equiv 0$ on $M_{2 L}$, and (iv) $|\nabla \beta| \leq 2 / L$. Given $\psi \in W$, let $\psi_{\text {in }}=(1-\beta) \psi$ and $\psi_{\text {ex }}=\beta \psi$. Note that the support of $\psi_{\text {in }}$ is contained in $M_{3 L}$, and the support of $\psi_{\mathrm{ex}}$ is contained in $\bigcup_{\alpha=1}^{\ell} E_{2 L}^{\alpha}$.

We have

$$
\begin{gathered}
\|\psi\|_{W}^{2}=\|\psi\|_{1,-1,2}^{2}=\left(\|\nabla \psi\|_{2}+\left\|\sigma^{-1} \psi\right\|_{2}\right)^{2} \leq 2\|\nabla \psi\|_{2}^{2}+2\left\|\sigma^{-1} \psi\right\|_{2}^{2}, \\
\left\|\sigma^{-1} \psi\right\|_{2}^{2}=\left\|\sigma^{-1} \psi\right\|_{2 ; M_{2 L}}^{2}+\sum_{\alpha=1}^{\ell}\left\|\sigma^{-1} \psi\right\|_{2 ; E_{2 L}^{\alpha}}^{2} .
\end{gathered}
$$

Recall that $\sigma \geq 1$, so

$$
\left\|\sigma^{-1} \psi\right\|_{2 ; M_{2 L}}^{2} \leq\|\psi\|_{2 ; M_{2 L}}^{2}=\left\|\psi_{\text {in }}\right\|_{2 ; M_{2 L}}^{2} \leq\left\|\psi_{\text {in }}\right\|_{2}^{2} .
$$

If $\psi \in C^{\infty}(M, S)$ and $\nabla \psi=0$, then $|\psi|$ is a constant. So $B(\psi, \phi)=\int_{M}\langle\nabla \psi, \nabla \phi\rangle$ is a positive definite Hermitian form on $C_{0}^{\infty}(M, S)$. We have

$$
\left\|\psi_{\text {in }}\right\|_{2}^{2} \leq c_{2}(L)\left\|\nabla \psi_{\text {in }}\right\|_{2}^{2}
$$

(see e.g. [25, Section 5.2]).

$$
\left\|\nabla \psi_{\text {in }}\right\|_{2}^{2}=\left\|\nabla \psi-\nabla \psi_{\text {ex }}\right\|_{2}^{2} \leq 2\|\nabla \psi\|_{2}^{2}+2\left\|\nabla \psi_{\text {ex }}\right\|_{2}^{2},
$$

where

$$
\left\|\nabla \psi_{\text {ex }}\right\|_{2}^{2}=\|\nabla(\beta \psi)\|_{2}^{2} \leq 2\|(\nabla \beta) \psi\|_{2}^{2}+2\|\beta \nabla \psi\|_{2}^{2} .
$$

Note that $\nabla \beta$ vanishes outside $M_{3 L} \backslash M_{2 L},|\nabla \beta| \leq 2 / L$ and $1 \leq 3 L \sigma^{-1}$ on $M_{3 L} \backslash M_{2 L}$, so

$$
\begin{gathered}
\|(\nabla \beta) \psi\|_{2}^{2} \leq\left\|\frac{2}{L} \psi\right\|_{2 ; M_{3 L} \backslash M_{2 L}}^{2} \leq\left\|\frac{2}{L} \cdot 3 L \cdot \sigma^{-1} \psi\right\|_{2 ; M_{3 L} \backslash M_{2 L}}^{2} \\
=36\left\|\sigma^{-1} \psi\right\|_{2 ; M_{3 L} \backslash M_{2 L}}^{2} .
\end{gathered}
$$

Recall that $|\beta| \leq 1$, so $\|\beta \nabla \psi\|_{2}^{2} \leq\|\nabla \psi\|_{2}^{2}$. So we have

$$
\begin{aligned}
\left\|\sigma^{-1} \psi\right\|_{2 ; M_{2 L}}^{2} & \leq\left\|\psi_{\text {in }}\right\|_{2}^{2} \leq c_{2}(L)\left\|\nabla \psi_{\text {in }}\right\|_{2}^{2} \leq 2 c_{2}(L)\left(\|\nabla \psi\|_{2}^{2}+\left\|\nabla \psi_{\text {ex }}\right\|_{2}^{2}\right) \\
& \leq 2 c_{2}(L)\left(3\|\nabla \psi\|_{2}^{2}+72\left\|\sigma^{-1} \psi\right\|_{2 ; M_{3 L} \backslash M_{2 L}}^{2}\right)
\end{aligned}
$$




$$
\begin{aligned}
\left\|\sigma^{-1} \psi\right\|_{2}^{2} & =\left\|\sigma^{-1} \psi\right\|_{2 ; M_{2 L}}^{2}+\sum_{\alpha=1}^{\ell}\left\|\sigma^{-1} \psi\right\|_{2 ; E_{2 L}^{\alpha}}^{2} \\
& \leq 6 c_{2}(L)\|\nabla \psi\|_{2}^{2}+\left(1+144 c_{2}(L)\right)\left(\sum_{\alpha=1}^{\ell}\left\|\sigma^{-1} \psi\right\|_{2 ; E_{2 L}^{\alpha}}^{2}\right) \\
& \leq 6 c_{2}(L)\|\nabla \psi\|_{2}^{2}+5\left(1+144 c_{2}(L)\right)\left(\sum_{\alpha=1}^{\ell}\|\nabla \psi\|_{2 ; E_{2 L}^{\alpha}}^{2}\right) \\
& \leq\left(726 c_{2}(L)+5\right)\|\nabla \psi\|_{2}^{2},
\end{aligned}
$$

where we used Lemma 22. So

$$
\|\psi\|_{W}^{2} \leq 2\|\nabla \psi\|_{2}^{2}+2\left\|\sigma^{-1} \psi\right\|_{2}^{2} \leq\left(1452 c_{2}(L)+12\right)\|\nabla \psi\|_{2}^{2} .
$$

By Lemma 20, $\|\nabla \psi\|_{2}^{2} \leq 20\|\mathbf{D} \psi\|_{2}^{2}$, so

$$
\|\psi\|_{W}^{2} \leq 240\left(121 c_{2}(L)+1\right)\|\mathbf{D} \psi\|_{2}^{2} .
$$

Lemma 24. For each $\eta \in C_{0}^{\infty}(N, S)$ there exists a unique $u \in W$ such that $\mathbf{D}^{2} u=$ $\eta$. $\psi=\mathbf{D} u \in W$ and

$$
\|\psi\|_{W}^{2} \leq c\|\eta\|_{2}^{2}
$$

Proof. Consider the functional

$$
F(u)=\frac{1}{2}\|\mathbf{D} u\|_{2}^{2}+\operatorname{Re}\langle u, \eta\rangle_{2}
$$

on $W$. It is strictly convex, weakly lower semicontinuous, and

$$
F(u) \geq \frac{1}{2 c}\|u\|_{W}^{2}-\|u\|_{W}\|\sigma \eta\|_{2},
$$

where $c$ is the constant in Lemma 23. By the calculus of variations, $F$ has a unique critical point $u \in W$ which is an absolute minimum in $W$, and $u$ is the weak solution to

$$
\mathbf{D}^{2} u=\eta
$$

Let $\psi=\mathbf{D} u \in W_{0}^{0,2}$. Then $\psi$ is a weak solution to

$$
\mathbf{D} \psi=\eta \text {. }
$$

By Lemma 17, $\psi \in W_{\text {loc }}^{1,2}(M, S) \cap C^{\infty}(M \backslash \partial \Omega, S)$. By Lemma 23.

$$
\|\psi\|_{W}^{2} \leq c\|\eta\|_{2}^{2} .
$$

So $\psi \in W$.

Lemma 25. For each $\eta \in C_{0}^{\infty}(N, S)$ there exists a unique $\psi \in W$ with $\mathbf{D} \psi=\eta$. For $p, \delta$ as in Lemma 19,

$$
\|\psi\|_{1, \delta, p} \leq c(\delta, p)\|\eta\|_{0, \delta+1, p} .
$$

Proof. By Lemma 24, there exists $\psi \in W$ such that $\mathbf{D} \psi=\eta$. The solution is unique by Lemma 23 .

By Lemma 17, $\psi \in W_{\text {loc }}^{1, p}(M, S) \cap C^{\infty}(M \backslash \partial \Omega, S)$. A slight modification of the proof of [27, Proposition 5.7] gives the a priori bound (57). 
The proof of [27, Proposition 5.9] gives

Proposition 26. Let $p, \delta$ be as in Lemma 19, Then $\mathbf{D}: W_{\delta}^{1, p} \rightarrow W_{\delta+1}^{0, p}$ is a surjection. If $\mathbf{D} \psi=\eta$, where $\eta \in W_{\delta}^{0, p}$ and $\psi \in W_{\delta}^{1, p}$, then the a priori bound (57) holds.

We now return to the proof of Theorem 8, Let $\psi_{0}$ be defined as in (49). Then $\mathbf{D} \psi_{0} \in L^{2}(M, S) \cap W_{1+\delta}^{0, p} \cap C^{\infty}(M, S)$ for all $2 \leq p<\infty$ and $0<\delta<1-3 / p$ (see [27, Section 4] for details). By Proposition 26, there exists $\psi_{1} \in W$ such that

$$
\mathbf{D} \psi_{1}=-\mathbf{D} \psi_{0}
$$

and $\psi_{1} \in W_{\delta}^{1, p}(M, S)$ for all $2 \leq p<\infty$ and $0<\delta<1-3 / p$. So

$$
\lim _{r \rightarrow \infty} r^{1-\epsilon}\left|\psi_{1}\right|=0
$$

for any $\epsilon>0$. By Lemma 17, $\psi \in C^{\infty}(M, S)$ and $\psi \in W_{\text {loc }}^{1, p}$ for any $2 \leq p<\infty$. Let $\psi=\psi_{1}+\psi_{0}$. Then $\psi$ is the desired solution in Theorem 8 . The solution is unique by Lemma 19 .

4.10. Proof of Theorem 7 . Let $\psi$ be the unique spinor given by Theorem 8 . By Proposition 10,

$$
0 \geq \frac{1}{10} \int_{M_{r}}|\nabla \psi|^{2}+\frac{1}{16} \int_{\Omega} u^{-2}|d u|^{2}|\psi|^{2}+\sum_{\alpha=1}^{\ell} \int_{S_{r}^{\alpha}}\left\langle\frac{H}{2} \psi-c(\nu) \check{\mathbf{D}} \psi, \psi\right\rangle .
$$

By calculations similar to those in [27, we have

$$
\lim _{r \rightarrow \infty} \int_{S_{r}^{\alpha}}\left\langle\frac{H}{2} \psi-c(\nu) \check{\mathbf{D}} \psi, \psi\right\rangle=-4 \pi G m_{\infty}^{\alpha}\left|\psi^{\alpha}\right|^{2},
$$

where $m_{\infty}^{\alpha}$ is the ADM mass of the end $E^{\alpha}$. So

$$
4 \pi G \sum_{\alpha=1}^{\ell} m_{\infty}^{\alpha}\left|\psi^{\alpha}\right|^{2} \geq \frac{1}{10} \int_{M}|\nabla \psi|^{2}+\frac{1}{16} \int_{\Omega} u^{-2}|d u|^{2}|\psi|^{2} .
$$

In particular, taking $\left|\psi^{\beta}\right|=1$ and $\psi^{\alpha}=0$ for $\alpha \neq \beta$, we have

$$
4 \pi G m_{\infty}^{\beta} \geq \frac{1}{10} \int_{M}|\nabla \psi|^{2}+\frac{1}{16} \int_{\Omega} u^{-2}|d u|^{2}|\psi|^{2} \geq 0 .
$$

If $m_{\infty}^{\beta}=0$ for some $\beta$, we have $d u=0$, so $u \equiv 1$, which implies $\hat{g}=\bar{g}$. We also have $\nabla \psi=0$ on $M$. We conclude that $\partial \Omega$ is connected and $M$ is the Euclidean space.

\section{ACKNOWLEDGMENTS}

We wish to thank Shi and Tam for their questions on 22] which motivated us to write down this proof in detail. We wish to thank Mu-Tao Wang for proofreading the first draft. The first author also wishes to thank Xiao Zhang for helpful discussions. 


\section{REFERENCES}

[1] R. Bartnik, New definition of quasilocal mass, Phys. Rev. Lett. 62 (1989), no. 20, 23462348. MR0996396 (90e:83041)

[2] R. Bartnik, Quasi-spherical metrics and prescribed scalar curvature, J. Differential Geom. 37 (1993), no. 1, 31-71. MR1198599(93i:53041)

[3] J.D. Brown and J.W. York, Quasilocal energy in general relativity, Mathematical aspects of classical field theory (Seattle, WA, 1991), 129-142, Contemp. Math., 132, Amer. Math. Soc., Providence, RI, 1992. MR.1188439

[4] J.D. Brown and J.W. York, Quasilocal energy and conserved charges derived from the gravitational action, Phys. Rev. D (3) 47 (1993), no. 4, 1407-1419. MR.1211109 (94h:83046)

[5] J.D. Brown, S.R. Lau and J.W. York, Energy of isolated systems at retarded times as the null limit of quasilocal energy, Phys. Rev. D (3) 55 (1997), no. 4, 1977-1984. MR1438691 (98b:83029)

[6] B. Booss-Bavnbek and K.P. Wojciechowski, Elliptic boundary problems for Dirac operators, Mathematics: Theory \& Applications, Birkhäuser Boston, Inc., Boston, MA, 1993. MR $1233386(94 \mathrm{~h}: 58168)$

[7] M.E. Cahill and G.C. McVittie, Spherical symmetry and mass-energy in general relativity. I. General theory, J. Mathematical Phys. 11 (1970), 1382-1391. MR0260376 (41:5003)

[8] D. Christodoulou and S.-T. Yau, Some remarks on the quasi-local mass, Mathematics and general relativity (Santa Cruz, CA, 1986), 9-14, Contemp. Math. 71, Amer. Math. Soc., Providence, RI, 1988. MR0954405 (89k:83050)

[9] A.J. Dougan and L.J. Mason, Quasilocal mass constructions with positive energy, Phys. Rev. Lett. 67 (1991), no. 16, 2119-2122. MR.1129012 (92g:83029)

[10] D.M. Eardley, Global problems in numerical relativity, Sources of gravitational radiation. Proceedings of the Workshop held at the Battelle Seattle Research Center, Seattle, Wash., July 24-August 4, 1978. Edited by Larry L. Smarr. Cambridge University Press, Cambridge-New York, 1979. MR0592635 (81j:83017)

[11] R.J. Epp, Angular momentum and an invariant quasilocal energy in general relativity, Phys. Rev. D 62 (2000), no. 12, 124108. MR.1814367 (2002a:83029)

[12] R. Geroch, Energy extraction, Ann. New York Acad. Sci. 224 (1973), 108-117.

[13] G.W. Gibbons, Collapsing shells and the isoperimetric inequality for black holes, Classical Quantum Gravity 14 (1997), 2905-2915. MR1476553 (98j:83056)

[14] D. Gilbarg and N.S. Trudinger, Elliptic partial differential equations of second order, Second edition, Grundlehren der mathematischen Wissenschaften [Fundamental Principles of Mathematical Sciences], 224. Springer-Verlag, Berlin, 1983. MR0737190 (86c:35035)

[15] S.W. Hawking, Gravitational radiation in an expanding universe, J. Math. Phys. 9 (1968), 598-604.

[16] S.W. Hawking and G.T. Horowitz, The gravitational Hamiltonian, action, entropy and surface terms, Classical Quantum Gravity 13 (1996), no. 6, 1487-1498. MR.1397130 (97d:83009)

[17] G. Huisken and T. Ilmanen, The inverse mean curvature flow and the Riemannian Penrose inequality, J. Differential Geom. 59 (2001), no. 3, 353-437. MR1916951 (2003h:53091)

[18] O. Hijazi, S. Montiel, and X. Zhang, Eigenvalues of the Dirac operator on manifolds with boundary, Comm. Math. Phys. 221 (2001), no. 2, 255-265. MR.1845323 (2002g:58051)

[19] P.S. Jang, On the positivity of energy in general relativity, J. Math. Phys. 19 (1978), no. 5, 1152-1155. MR0488515 (80b:83012a)

[20] J. Kijowski, A simple derivation of canonical structure and quasi-local Hamiltonians in general relativity, Gen. Relativity Gravitation 29 (1997), no. 3, 307-343. MR1439857 (97m:83029)

[21] S.R. Lau, New Variables, the gravitational action and boosted quasilocal stress-energymomentum, Classical Quantum Gravity 13 (1996), 1509-1540. MR.1397132 (97d:83037)

[22] C.-C. M. Liu and S.-T. Yau, Positivity of quasilocal mass, Phys. Rev. Lett. 90 (2003), no. 23, 231102. MR2000281 (2004g:83031)

[23] M. Ludvigsen and J.A.G. Vickers, Momentum, angular momentum and their quasilocal null surface extensions, J. Phys. A 16 (1983), no. 6, 1155-1168. MR0706691 (85g:83014)

[24] H. Minkowski, Volumen und Oberfläche, Math. Ann. 57, 447-495. 
[25] C. B. Morrey, Jr., Multiple integrals in the calculus of variations, Die Grundlehren der mathematischen Wissenschaften, Band 130, Springer-Verlag New York, Inc., New York, 1966. MR0202511 (34:2380)

[26] N. Ó Murchadha, L.B. Szabados, K.P. Tod, Comment on "Positivity of quasilocal mass", Phys. Rev. Lett. 92 (2004), No. 25, 259001. MR2114434

[27] T. Parker, C.H. Taubes, On Witten's proof of the positive energy theorem, Comm. Math. Phys. 84 (1982), no. 2, 223-238. MR0661134 (83m:83020)

[28] R. Penrose, Quasilocal mass and angular momentum in general relativity, Proc. R. Soc. Lond. A385 (1982), 53-63. MR0661716 (83j:83024)

[29] R. Penrose, W. Rindler, Spinors and space-time, Vol. 2, Spinor and twistor methods in space-time geometry, Second edition, Cambridge Monographs on Mathematical Physics, Cambridge University Press, Cambridge, 1988. MR0944085 (89d:83010)

[30] A.V. Pogorelov, Some results on surface theory in the large, Advances in Math. 1 (1964), fasc. 2, 191-264. MR.0178438 (31:2695)

[31] Y. Shi and L.-F. Tam, Positive mass theorem and the boundary behaviors of compact manifolds with nonnegative scalar curvature, J. Differential Geom. 62 (2002), no. 1, 79125. MR.1987378 (2005b:53046)

[32] R. Schoen and S.-T. Yau, Proof of the positive mass theorem II, Comm. Math. Phys. 79 (1981), no. 2, 231-260. MR0612249 (83i:83045)

[33] L.B. Szabados, Quasi-local energy-momentum and angular momentum in GR: a review article, Living Rev. Relativity, 7 (2004), 4.

[34] K.P. Tod, More on Penrose's quasi-local mass, Classical Quantum Gravity 3 (1986), no. 6, 1169-1189. MR0868724 (88i:83049)

[35] E. Witten, A new proof of the positive energy theorem, Comm. Math. Phys. 80 (1981), no. 3, 381-402. MR0626707 (83e:83035)

[36] S.-T. Yau, Geometry of three manifolds and existence of black hole due to boundary effect, Adv. Theor. Math. Phys. 5 (2001), no. 4, 755-767. MR1926294(2003j:53052)

Department of Mathematics, Harvard University, Cambridge, Massachusetts 02138 E-mail address: ccliu@math.harvard.edu

Current address: Department of Mathematics, Northwestern University, Evanston, Illinois 60208

E-mail address: ccliu@math.northwestern.edu

Department of Mathematics, Harvard University, Cambridge, Massachusetts 02138

E-mail address: yau@math.harvard.edu 